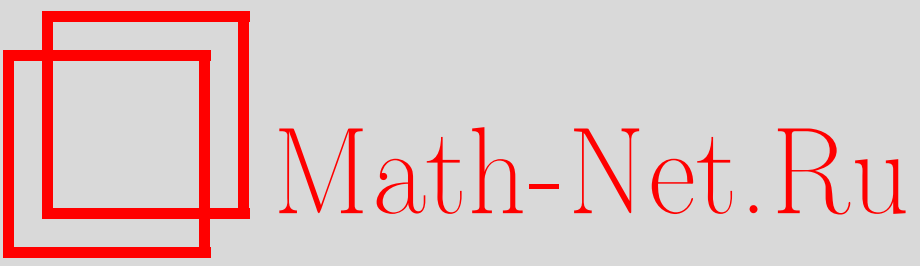

А. Н. Капустин, Д. О. Орлов, Лекции о зеркальной симметрии, производных категориях и $D$-бранах, $У M H, 2004$, том 59, выпуск 5, 101-134

DOI: https://doi.org/10.4213/rm772

Использование Общероссийского математического портала Math-Net.Ru подразумевает, что вы прочитали и согласны с пользовательским соглашением

http://www.mathnet.ru/rus/agreement

Параметры загрузки:

IP: 52.90 .164 .192

26 апреля 2023 г., 14:19:39 


\title{
ЛЕКЦИИ О ЗЕРКАЛЬНОЙ СИММЕТРИИ, ПРОИЗВОДНЫХ КАТЕГОРИЯХ И $D$-БРАНАХ
}

\author{
А.Н. КАПУСТИН, Д. О. ОрлОВ
}

\begin{abstract}
Данная статья, рассчитанная, в первую очередь, на математическую аудиторию, представляет из себя введение в гомологическую зеркальную симметрию, производные категории и топологические $D$-браны. В статье объясняется зеркальная симметрия с физической точки зрения, ее связь с производными категориями и причина, по которой категория Фукая должна быть расширена с помощью коизотропных $A$-бран, обсуждается, как распространить определение гомологий Флоера на такие объекты и дается описание зеркальной симметрии для плоских торов. Статья состоит из четьрех лекций, которые были прочитаны в Институте чистой и прикладной математики (Лос-Анджелес) в марте 2003 г. в рамках программы "Симплектическая геометрия и физика".

Библиография: 47 названий.
\end{abstract}

\section{СОДЕРЖАНИЕ}

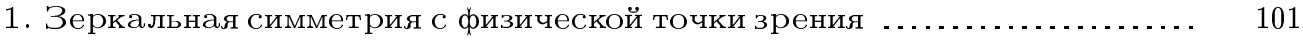

2. Зеркальная симметрия для плоских комплексных торов ............. 112

3. Производные категории когерентных пучков и тестирование гипотезы

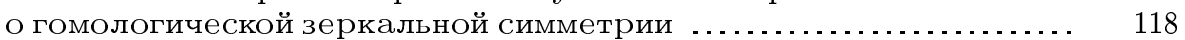

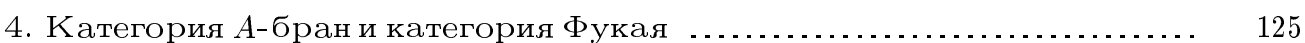

Список литературы ................................................. 132

\section{1. Зеркальная симметрия с физической точки зрения}

Цель данной лекщии - объяснить феномен зеркальной симметрии с физической точки зрения, а также его математическую интерпретацию, выдвинутую М. Концевичем в 1994 г. в докладе на Международном Математическом Конгрессе [25]. Другой подход к зеркальной симметрии был предложен в работе А. Стромингера, Ш. Т. Яу и Э. Заслоу [41]; здесь он обсуждаться не будет.

С физической точки зрения, зеркальная симметрия - это некоторое соответствие на множестве $2 d$-конформных теорий поля с $N=2$ суперсимметрией. А $2 d$-конформная

Первьй автор поддержан фондом DOE (грант DE-FG03-92-ER40701). Второй автор поддержан РФФИ (грант 02-01-00468), грантом Президента РФ для поддержки молодых российских ученых МД-2731.2004.1, Американским фондом гражданских исследований CRDF RM1-2405МО-02, Фондом содействия отечественной науке. 
теория поля является довольно сложной структурой алгебраической природы; некоторое понятие об этой структуре будет дано ниже. Таким образом, зеркальная симметрия - тоже понятие алгебраическое. Геометрия возникнет, когда мы рассмотрим специальный класс суперконформных теорий поля, связанный с многообразиями Калаби-Яу.

Начнем с $2 d$-конформной теории поля (КТП). Чтобы задать КТП, нужны следуюшие данные: бесконечномерное векторное пространство $V$ (пространство состояний), три выделенных элемента в $V$ (вакуумный вектор $|v a c\rangle$ и еще два элемента $L, \bar{L}$ ) и линейное отображение $Y$ из $V$ в "пространство формальных дробных степенных рядов по $z, \bar{z}$ с коэффициентами в $\operatorname{End}(V)$ ". Мы будем назьвать отображение $Y$ операторно-векторным соответствием. Точное определение "пространства формальных дробных степенных рядов" было дано в [19]. Для простоты можно считать, что $Y$ принимает значения в пространстве рядов Лорана по $z, \bar{z}$ с коэффициентами в $\operatorname{End}(V)$, хотя такое определение $Y$ недостаточно общее в контексте зеркальной симметрии. Эти данные должны удовлетворять аксиомам, аккуратную формулировку которых можно найти в [19]. На интуитивном уровне аксиомы вьглядят следуюшим образом.

(i) $Y(|v a c\rangle)=\mathrm{id}_{V}$.

(ii) $Y(L)=\sum_{n \in \mathbb{Z}} L_{n} / z^{n+2}, Y(\bar{L})=\sum_{n \in \mathbb{Z}} \bar{L}_{n} / \bar{z}^{n+2}$ для некоторых $L_{n}, \bar{L}_{n} \in$ $\operatorname{End}(V)$.

(iii) $\mathrm{KaK} L_{n}$, так и $\bar{L}_{n}$ удовлетворяют коммутационным соотношениям алгебры Вирасоро; все $L_{n}$ коммутируют со всеми $\bar{L}_{m}$.

(iv) Имеют место соотношения $\left[L_{-1}, Y(v, z, \bar{z})\right]=\partial Y(v, z, \bar{z}),\left[L_{0}, Y(v, z, \bar{z})\right]=$ $z \partial Y(v, z, \bar{z})+Y\left(L_{0} v, z, \bar{z}\right)$, для любого $v \in V$ и аналогичные соотношения, получающиеся заменой $L_{n} \rightarrow \bar{L}_{n}, \partial \rightarrow \bar{\partial}$.

(v) $Y(v)|v a c\rangle=e^{z L_{-1}+\bar{z} \bar{L}_{-1}} v$ для любого $v \in V$.

(vi) $Y(v, z, \bar{z}) Y\left(v^{\prime}, z^{\prime}, \bar{z}^{\prime}\right)$ имеет не более чем степенные сингулярности на диагоналях $z=z^{\prime}$ и $\bar{z}=\bar{z}^{\prime}$.

(vii) $Y(v, z, \bar{z}) Y\left(v^{\prime}, z^{\prime}, \bar{z}^{\prime}\right)-Y\left(v^{\prime}, z^{\prime}, \bar{z}^{\prime}\right) Y(v, z, \bar{z})$ является формальным распределением с носителем на диагонали.

Напомним, что алгебра Вирасоро - это бесконечномерная алгебра Ли с генераторами $L_{m}, m \in \mathbb{Z}$, и следующими коммутационньми соотношениями:

$$
\left[L_{m}, L_{n}\right]=(m-n) L_{m+n}+c \frac{m^{3}-m}{12} \delta_{m,-n} .
$$

Она является центральным расширением алгебры Витта (алгебры Ли векторных полей на окружности). Константа $c$ назьвается центральным зарядом. Алгебра Вирасоро, натянутая на $L_{n}$ (или $\left.\bar{L}_{n}\right)$, назьвается правобегущей (соответственно левобегущей).

Сушествует несколько вариантов вьшеприведенного определения КТП. Для наших целей необходимо сделать векторные пространства и отображения между ними $(\mathbb{Z} / 2)$-градуированными. С физической точки зрения это означает, что в КТП есть как бозонные, так и фермионные поля. Другое важное свойство, вьполненное в любой приемлемой КТП, - это сушествование невырожденной билинейной формы на $V$, согласованной с остальными структурами КТП. Наконец, большинство КТП, рассматриваемых в физической литературе, обладает лево-правой симметрией. Это означает, что замена $z$ на $\bar{z}$ и $L_{n}$ на $\bar{L}_{n}$ дает изоморфную КТП. Мы будем рассматривать только лево-право симметричные КТП. 
Более геометричное определение КТП было дано Г.Б. Сигалом в работе [40]. Сигал рассматривал категорию, объектами которой являются конечные наборы окружностей, а морфизмами - римановы поверхности с ориентированньми аналитически-параметризованными границами. Композиция морфизмов определена посредством склеивания римановых поверхностей вдоль граничных окружностей с согласованной ориентацией. КТП определяется как проективньй функтор из этой категории в категорию гильбертовых пространств, удовлетворяюший набору аксиом, описанных в работах $[40],[10]$. (Проективньй функтор из категории $\mathscr{C}$ в категорию гильбертовых пространств - это функтор из $\mathscr{C}$ в категорию, объектами которой являются гильбертовы пространства, а морфизмами - классы эквивалентности морфизмов гильбертовых пространств по отношению к операции умножения на ненулевые скаляры.) Можно показать, что любая КТП в смысле Сигала дает КТП в смысле нашего алгебраического определения (см. [10]). Например, векторное пространство $V$ в нашем определении это гильбертово пространство, соответствующее одной окружности. Отображение $Y$ получается из рассмотрения морфизма гильбертовых пространств, соответствующего римановой сфере с тремя дырками. Похоже, что, и обратно, по любой КТП в нашем смысле, обладаюшей лево-правой симметрией и согласованным скалярным произведением на $V$, можно однозначно восстановить сигаловскую КТП в роде нуль (т.е. ограничиваясь римановыми поверхностями рода нуль).

$N=1$ супералгебра Вирасоро - это некая бесконечномерная супералгебра Ли, содержашая в качестве подалгебры алгебру Вирасоро. Кроме четных генераторов Вирасоро $L_{n}, n \in \mathbb{Z}$, она содержит также нечетные генераторы $Q_{n}, n \in \mathbb{Z}$. Новые коммутационные соотношения вьглядят так:

$$
\left[L_{m}, Q_{n}\right]=\left(\frac{m}{2}-n\right) Q_{m+n}, \quad\left[Q_{m}, Q_{n}\right]=\frac{1}{2} L_{m+n}+\frac{c}{12} m^{2} \delta_{m,-n} .
$$

По определению $N=1$ суперконформная теория поля (СКТП) - это такая КТП, где на $V$ задано представление двух копий $N=1$ супералгебры Вирасоро, и это представление согласовано с другими структурами КТП в очевидном смысле. ${ }^{1}$

$N=2$ супералгебра Вирасоро - дальнейшее обобщение алгебры Вирасоро. Это бесконечномерная супералгебра Ли, содержащая в качестве подалгебры $N=1 \mathrm{cy-}$ пералгебру Вирасоро. Ее четные генераторы обозначаются $L_{n}, J_{n}, n \in \mathbb{Z}$. Нечетные генераторы обозначаются $Q_{n}^{+}, Q_{n}^{-}, n \in \mathbb{Z}$. Коммутационные соотношения имеют следуюшую структуру:

$$
\begin{gathered}
{[L, L] \sim L, \quad[J, J] \sim \text { central, },[L, J] \sim J, \quad\left[Q^{ \pm}, Q^{ \pm}\right]=0} \\
{\left[L, Q^{ \pm}\right] \sim Q^{ \pm}, \quad\left[J, Q^{ \pm}\right] \sim \pm Q^{ \pm}, \quad\left[Q^{ \pm}, Q^{\mp}\right] \sim J+L+\text { central } .}
\end{gathered}
$$

Точный вид коммутационных соотношений можно найти, например, в работе [19]. Вьшеупомянутая $N=1$ подалгебра Вирасоро натянута на элементы $L_{n}$ и $Q_{n}=$ $Q_{n}^{+}+Q_{n}^{-}, n \in \mathbb{Z}$. Отношения между $N=1$ и $N=2$ супералгебрами Вирасоро аналогичны отношениям между дифференщиалами де Рама и Дольбо на формах на комплексном многообразии: генераторы $Q_{n}$ аналогичны дифференциалу де Рама $d$, в то время как $Q_{n}^{+}$и $Q_{n}^{-}$аналогичны дифференциалам Дольбо $\partial$ и $\bar{\partial}$.

\footnotetext{
${ }^{1}$ Строго говоря, это определение относится к сектору Рамона-Рамона СКТП.
} 
$N=2$ CКТП - это $N=1$ СКТП с заданным действием на $V$ двух копий $N=2$ супералгебры Вирасоро. Это действие должно быть согласовано с остальными структурами СКТП. Таким образом, мы получаем иерархию алгебраических структур:

множество КТП つ множество $N=1$ СКТП つ множество $N=2$ СКТП.

Существует еще более общее понятие: $2 d$-теория поля, безприлагательного "конформная". Мы его обсуждать не будем.

Можно дать более геометричное определение $N=1$ и $N=2$ СКТП в стиле Сигала. Для этого нужно заменить римановы поверхности двумерными супермногообразиями с $N=1$ или $N=2$ суперконформной структурой.

Изоморфизм КТП или СКТП - это изоморфизм векторных пространств $V \stackrel{\sim}{\rightarrow} V^{\prime}$, который согласован со всеми остальньми структурами в очевидном смысле. Две $N=2$ CKТП могут быть изоморфны как $N=1$ CKTП, не будучи изоморфны как $N=2$ СКТП. (Заметим, что в физической литературе при обсуждении изоморфизмов СКТП часто не указано явно, имеются в виду $N=1$ или $N=2$ изоморфизмы: обычно это ясно из контекста.)

$N=2$ супералгебра Вирасоро имеет интересньй автоморфизм, назьваемьй зеркальным автоморфизмом:

$$
\mathfrak{M}: L_{n} \mapsto L_{n}, \quad J_{n} \mapsto-J_{n}, \quad Q_{n}^{ \pm} \mapsto Q_{n}^{\mp}
$$

Пусть у нас имеется пара $N=2$ CКТП, которые изоморфны как $N=1$ CKТП, и пусть $f: V \stackrel{\sim}{\rightarrow} V^{\prime}$ - некоторый изоморфизм между ними. Мы будем говорить, что $f-$ правый зеркальньй морфизм между нашими $N=2$ CKTП, если $f$ действует как единичньй автоморфизм на левобегущую $N=2$ супералгебру Вирасоро и как зеркальньй автоморфизм на правобегущую $N=2$ супералгебру Вирасоро. Это определение имеет смысл, поскольку, как замечено выше, зеркальньй автоморфизм тривиально действует на $N=1$ подалгебру Вирасоро. Переставляя левое и правое, получаем определение левого зеркального морфизма между $N=2$ СКТП. Наконец, если $f$ действует на обе $N=2$ супералгебры Вирасоро как зеркальный автоморфизм, мы будем говорить, что $f$ - это комплексное сопряжение пространства значений.

Назовем две $N=2$ СКТП зеркальными друг другу, если сушествует левый или правый зеркальный морфизм между ними. Очевидно, что если две $N=2$ CKTП зеркальны третьей СКТП (слева), то эти две СКТП сами изоморфны (как $N=2$ СКТП). Таким образом, отношение зеркальности (скажем, слева) - это инволютивное отношение на множестве классов изоморфизмов $N=2$ CКТП. Подчеркнем, что зеркальные $N=2$ СКТП изоморфны как $N=1$ CКТП, но обычно неизоморфны как $N=2$ СКТП. В физической литературе построено много явных примеров зеркальных пар $N=2$ СКТП; из них можно строить более сложные примеры посредством операций тензорного произведения, орбифолда и т. д.

Рассмотрим теперь связь между $N=2$ СКТП и многообразиями Калаби-Яу. Физическое многообразие Калаби-Яу - это компактное комплексное многообразие с тривиальным каноническим классом, снабженное кэлеровым классом и В-полем (элементом групшы $H^{2}(X, \mathbb{R}) / H^{2}(X, \mathbb{Z})$ ). Гипотетически, каждому физическому многообразию Калаби-Яу можно естественным образом сопоставить $N=2$ СКТП, зависящую 
от этих геометрических данных. Если не гнаться за строгостью, то процедуру построения $N=2$ СКТП можно описать так. Во-первых, каждому физическому многообразию Калаби-Яу легко сопоставить классическую теорию поля, назьваемую $N=2$ сигма-моделью. Ее лагранжиан дается явной, хотя и довольно сложной, формулой (см., например, [36]). Этот лагранжиан инвариантен относительно большой супералгебры инфинитезимальных преобразований, которая изоморфна сумме двух копий $N=2$ супералгебры Вирасоро (с нулевьм центральным зарядом). Во-вторых, надо проквантовать $N=2$ сигма-модель таким образом, чтобы $N=2$ суперконформная симметрия сохранялась и на квантовом уровне (при этом неизбежно появляется ненулевой центральньй заряд). Результат квантования и есть искомая $N=2$ СКТП.

За исключением нескольких очень специальных случаев, точно проквантовать сигма-модель не удается, и приходится прибегать к теории возмушений. А именно, метрика умножается на положительное число $t \gg 1, g_{\mu \nu} \rightarrow t^{2} g_{\mu \nu}$, рассматривается предел $t \rightarrow \infty$ (так назьваемый предел большого объема) и квантование проводится, используя разложение по степеням $1 / t$. Гипотетически, получающиеся степенные ряды по $1 / t$ имеют ненулевой радиус сходимости и тем самым определяют $N=2$ СКТП.

Естественно поставить вопрос, можно ли восстановить физическое многообразие Калаби-Яу $X$ по $N=2$ СКТП. Мы вскоре увидим, что однозначно этого сделать нельзя. Тем не менее, довольно просто определить некоторые численные характеристики $X$. Например, комплексная размерность $X$ равна $c / 3$, где $c$ - это центральный заряд $N=2$ супералгебры Вирасоро. Числа Ходжа $h^{p, q}(X)$ можно вычислить следуюшим образом. Из коммутационных соотношений $N=2$ супералгебры Вирасоро следует, что оператор $D_{B}=Q_{0}^{+}+\bar{Q}_{0}^{+}$, действующий на пространстве $V$, удовлетворяет $D_{B}^{2}=0$. Оператор $D_{B}$ назьвается БРСТ-оператором (типа $B$, см. ниже), а его когомологии назьвается БРСТ-когомологиями. Для любой разумной $N=2 \mathrm{CKT \Pi}$ когомологии $D_{B}$ конечномерны и биградуированы собственными значениями операторов $J_{0}$ и $\bar{J}_{0}$. (Эти операторы назьваются левобегушим и правобегушим $R$-зарядами соответственно.) Число Ходжа $h^{p, q}(X)$ равно размерности компоненты БРСТ когомологии с $R$-зарядами $p-n / 2$ и $q-n / 2$, где $n=\operatorname{dim}_{\mathbb{C}} X$. Отсюда ясно, что не любая $N=2$ СКТП имеет интерпретацию в терминах многообразий Калаби-Яу: необходимьм условием существования такой интерпретации является целочисленность $n=c / 3$ и целочисленность спектра операторов $J_{0}+n / 2$ и $\bar{J}_{0}+n / 2$ на БРСТ-когомологии. Гипотетически, необходимым и достаточньм условием существования геометрической интерпретации $N=2$ СКТП является целочисленность $n=c / 3$ и целочисленность спектра операторов $J_{0}+n / 2$ и $\bar{J}_{0}+n / 2$ на $V$. При этом необходимо несколько расширить понятие многообразия Калаби-Яу, включив, например, некоторые орбифолды.

Можно показать, что если два физических многообразияхш Калаби-Яу связаны комплексным сопряжением, то соответствуюшие $N=2$ СКТП связаны комплексным сопряжением пространства значений (см. вьше). Этим и объясняется выбор терминологии. Обратное неверно: сушествование морфизма между двумя $N=2$ CKТП, которьй является $N=1$ изоморфизмом и индуцирует зеркальньй автоморфизм на обеих копиях $N=2$ супералгебры Вирасоро, не влечет никакой простой связи между соответствуюшими многообразиями Калаби-Яу. Напрашивается следуюший вопрос.

Вопрос 1. При каких условиях два многообразия Калаби-Яу дают изоморфные $N=2$ CKTП?

Чтобы ответить на этот вопрос, нужно дать геометрическую интерпретацию 
“квантовьм симметриям" $N=2$ CKТП. Вот еще один вопрос такого же типа.

Вопрос 2. При каких условиях два многообразия Калаби-Яу дают $N=2$ CКТП, связанные зеркальным морфизмом?

Мы будем говорить, что два физических многообразия Калаби-Яу зеркальны друг

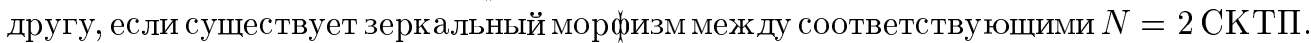

Первые нетривиальные примеры зеркальных многообразий Калаби-Яу были построены в работе Б. Грина и Р. Плессера [14]. В комплексной размерности три (наиболее интересной с физической точки зрения) простейший нетривиальньй пример имеет следуюший вид. Первое многообразие Калаби-Яу - это квинтика Ферма в $\mathbb{C P}^{4}$, заданная уравнением $x^{5}+y^{5}+z^{5}+v^{5}+w^{5}=0$. Чтобы построить зеркальное ей многообразие Калаби-Яу, нужно взять ту же самую квинтику, профакторизовать ее по некоторому действию группы $(\mathbb{Z} / 5)^{3}$ и раздуть неподвижные точки. Мы не будем здесь объяснять, почему эта пара многообразий зеркальна друг другу. (В статье Грина и Плессера это было выведено из гипотетической эквивалентности $N=2 \mathrm{CKT \Pi ,}$ соответствующей квинтике Ферма, и некоторой точно решаемой $N=2$ CKТП, построенной Д. Гепнером. В последствии эта эквивалентность была изучена Э. Виттеном [44], так что в настояшее время она рассматривается физиками как теорема.)

Ответ на первьй вопрос оказьвается весьма нетривиальным. Это видно уже на примере, в котором $X$ - это комплексный тор с плоской метрикой (см. лекцию 2). Например, тор и его дуальный тор соответствуют одной и той же $N=2$ СКТП, хотя они обычно не изоморфны как комплексные многообразия. Что касается вопроса 2 характеризации зеркального соответствия в геометрических терминах, - то ответ на него и есть конечная цель всех исследований по зеркальной симметрии.

На самом примитивном уровне, зеркальная симметрия между $X$ и $X^{\prime}$ влечет связь между числами Ходжа $h^{p, q}(X)$ и $h^{p, q}\left(X^{\prime}\right)$. Чтобы понять, как это происходит, заметим, что наряду с когомологией оператора $D_{B}=Q_{0}^{+}+\bar{Q}_{0}^{+}$можно также рассмотреть когомологию оператора $D_{A}=Q_{0}^{-}+\bar{Q}_{0}^{+}$. Можно показать, что если вьполнены условия целочисленности на спектр $J_{0}, \bar{J}_{0}$, упомянутые выше, то эти две когомологии изоморфны как биградуированные векторные пространства [29]. Далее, если $X$ и $X^{\prime}$ зеркальны, то когомология $D_{A}(X)$ бистепени $(\alpha, \beta)$ изоморфна когомологии $D_{B}\left(X^{\prime}\right)$ бистепени $(-\alpha, \beta)$. Используя связь когомологии $D_{B}(X)$ с числами Ходжа многообразия $X$, легко видеть, что

$$
h^{p, q}(X)=h^{n-p, q}\left(X^{\prime}\right) .
$$

Если выписать числа Ходжа на плоскости с координатами $p-q$ и $p+q-n$, то получится таблица в форме ромба (ромб Ходжа). Для любого кэлерова многообразия этот ромб переходит в себя при повороте на $180^{\circ}$. Уравнение (3) означает, что ромбы Ходжа зеркальных многообразий связаны поворотом на $90^{\circ}$.

Конечно, зеркальная симметрия не сводится к числам Ходжа. Наиболее перспективньй подход к проблеме характеризации зеркальной симметрии с геометрической точки зрения был предложен М. Концевичем в 1994 г. В оставшейся части этой лекции мы обрисуем в обших чертах гипотезу Конщевича, а также ее физическую интерпретацию.

Физическое многообразие Калаби-Яу обладает как комплексной, так и симплектической структурой (кэлеровой формой). Чтобы прояснить геометрический смысл зеркальной симметрии, очень полезно "расцепить" эти два типа геометрических структур и сфокусироваться на одной из них. Точнее говоря, кэлерову форму 
надо объединить с $(1,1)$-компонентой В-поля в “комплексифицированную кэлерову форму", которая параметризует "расширенное пространство модулей симплектических структур". Аналогично, нужно считать, что комплексная структура вместе с $(0,2)$-частью В-поля параметризуют "расширенное пространство модулей комплексных структур". Мы хотели бы выделить те аспекты $N=2$ CKTП, которые зависят только от расширенных комплексных или только от расширенных симплектических модулей. Этого можно достигнуть, воспользовавшись приемом, изобретенньм Э. Виттеном [45], [46] и называемым “топологическим скручиванием”.

Суть конструкции Виттена заключается в том, что во многих $N=2$ СКТП есть конечномерные секторы, которые являются топологическими теориями поля, т.е. они не зависят от двумерной метрики (метрики на "мировом листе", говоря на языке теории струн). На самом деле, во многих $N=2$ CKТП, включая СКТП, связанные с многообразиями Калаби-Яу, есть два таких сектора, которые называются $A$ - и $B$-моделями.

Напомним основные сведения про двумерные топологические теории поля $(2 d$ ТTП). Эти теории определяются аксиомами, похожими на сигаловские аксиомы конформной теории поля, но значительно более простыми [2]. Рассмотрим категорию, объектами которой являются конечные наборы ориентированных параметризованных окружностей, а морфизмами - ориентированные двумерные многообразия с гранищей (не снабженные комплексной структурой). $2 d$-ТТП - это функтор из этой категории в категорию конечномерных градуированных пространств, удовлетворяющий набору свойств, аналогичных сигаловским. Как и для двумерных КТП, это определение может быть переформулировано в чисто алгебраических терминах. Оказьвается, что понятие ТТП полностью эквивалентно понятию суперкоммутативной фробениусовой алгебры, т.е. суперкоммутативной алгебры с инвариантной метрикой (см., например, [6]).

Подробное описание процедуры топологического скручивания увело бы нас слишком далеко. Грубо говоря, надо “урезать” пространство $V$ до когомологии некоторого оператора, действующего на $V$ (этот оператор обычно назьвают БРСТ-оператором). Операторно-векторное соответствие на $V$ дает структуру суперкоммутативной алгебры на БРСТ-когомологиях, в то время как невырожденное скалярное произведение на $V$ индуцирует невырожденное скалярное произведение на БРСТ-когомологиях.

Есть два существенно разных БРСТ-оператора: $D_{A}$ или $D_{B}$. (Можно также рассмотреть БРСТ-операторы $D_{A}^{\prime}=Q_{0}^{+}+\bar{Q}_{0}^{-}$и $D_{B}^{\prime}=Q_{0}^{-}+\bar{Q}_{0}^{-}$, но это не дает ничего нового, поскольку замена $X$ на его комплексно сопряженное переводит $D_{A}^{\prime}$ в $D_{A}$, а $D_{B}^{\prime}$ в $\left.D_{B}.\right)$ Таким образом, конструкция Виттена сопоставляет каждому физическому многообразию Калаби-Яу две ТТП, которые назьваются $A$-моделью и $B$-моделью соответственно.

Можно показать, что $A$-модель не зависит от "расширенной комплексной структуры” на $X$, а $B$-модель не зависит от "расширенной симплектической структуры” на $X$. Иначе говоря, $A$-модель - это симплектический инвариант Калаби-Яу, а $B$-модель комплексный. На самом деле, пространство состояний $A$-модели естественным образом изоморфно когомологиям де Рама $H^{*, *}(X)$, в то время как пространство состояний $B$-модели естественным образом изоморфно когомологиям Дольбо

$$
H^{*}\left(\Lambda^{*} T^{1,0} X\right)
$$

где $T^{1,0} X$ - это голоморфное касательное расслоение $X$. Поскольку каноническое линейное расслоение $X$ тривиально, пространство $H^{q}\left(\Lambda^{p} T^{1,0} X\right)$ изоморфно простран- 
ству $H^{n-p, q}(X)$; этот изоморфизм зависит от выбора тривиализации канонического расслоения. Заметим также, что оба пространства состояний имеют естественную биградуировку. С физической точки зрения, она возникает благодаря наличию $R$-зарядов $J_{0}$ и $\bar{J}_{0}$.

Как уже говорилось вьше, пространство состояний ТТП имеет структуру суперкоммутативной алгебры. В случае $B$-модели это очевидная структура; в случае $A$-модели - это деформация очевидной структуры, зависяшая от "расширенной симплектической структуры" на $X$ : так называемые квантовые когомологии $X$.

Зеркальная симметрия действует на $A$ - и $B$-модели очень простым образом. Легко видеть, что зеркальньй автоморфизм супералгебры Вирасоро переставляет $D_{A}$ и $D_{B}$. Таким образом, если у нас есть зеркальная пара физических многообразий Калаби-Яу $X$ и $X^{\prime}$, то $A$-модель для $X$ изоморфна $B$-модели для $X^{\prime}$, и наоборот. Если $A$-модель для $X$ изоморфна $B$-модели для $X^{\prime}$ и наоборот, мы будем говорить, что $X$ и $X^{\prime}$ слабо-зеркальны друг другу. С понятием слабой зеркальной симметрии гораздо легче работать, чем с "настояшей" зеркальной симметрией, поскольку ее можно проверить, не зная ничего про сигма-модели и их квантование. С другой стороны, в процессе топологического скручивания теряется много информации, и возникает желание найти “обогашенный” вариант $A$ - и $B$-моделей, который был бы ближе по информационному содержанию к $N=2$ CKТП.

М. Конщевич предложил рассмотреть в качестве такого “обогашения" $B$-модели ограниченную производную категорию когерентных пучков на $X$, которая обозначается $\mathbf{D}^{b}(X)$; аналогично, для $A$-модели он предложил рассмотреть производную версию так называемой категории Фукая, которую мы будем обозначать $\mathbf{D} \mathscr{F}_{0}(X)$. Другими словами, гипотеза Концевича заключается в том, что если $X$ и $X^{\prime}$ зеркальны, то категория $\mathbf{D}^{b}(X)$ эквивалентна категории $\mathbf{D} \mathscr{F}_{0}\left(X^{\prime}\right)$, и наоборот. Это так называемая гипотеза гомологической зеркальной симметрии (ГЗС). Если обратное утверждение тоже верно, то это давало бы полный ответ на вопросы 1 и 2.

Обрисуем теперь в общих чертах определения этих двух категорий. Пусть $X$ гладкое комплексное многообразие. Объект $\mathbf{D}^{b}(X)$ - это ограниченньй комплекс голоморфных векторных расслоений на $X$, т.е. конечная последовательность голоморфных векторных расслоений и отображений между ними

$$
0 \rightarrow \cdots \rightarrow E_{n-1} \rightarrow E_{n} \rightarrow E_{n+1} \rightarrow \cdots \rightarrow 0
$$

такая, что композиция любых двух последовательных отображений равна нулю. Напомним, что когомология такого комплекса - это последовательность когерентных пучков на $X$. Чтобы определить морфизмы в $\mathbf{D}^{b}(X)$, рассмотрим сначала категорию ограниченных комплексов $\mathbf{C}^{b}(X)$, где объекты - такие же, как в $\mathbf{D}^{b}(X)$, а морфизмыцепные отображения комплексов. Морфизм в категории $\mathbf{C}^{b}(X)$ назьвается квазиизоморфизмом, если он индуцирует изоморфизм на когомологиях. Смысл определения производной категории в том, чтобы отождествить квазиизоморфные комплексы. То есть $\mathbf{D}^{b}(X)$ получается из $\mathbf{C}^{b}(X)$ формальньм присоединением обратных морфизмов ко всем квазиизоморфизмам. В этом определении можно заменить комплексы голоморфных расслоений комплексами произвольных когерентных пучков: производная категория от этого не меняется. В лекщии 3 мы обсудим $\mathbf{D}^{b}(X)$ более подробно.

В то время как когерентные пучки и их комплексы давно стали одним из основных понятий алгебраической геометрии, производная категория Фукая - недавнее изобретение. Она получается из некой геометрической категории, назьваемой категорией 
Фукая $\mathscr{F}(X)$, посредством довольно сложной алгебраической конструкции. Данная категория была введена К. Фукая в [8]. На самом деле, это не совсем категория: на пространствах морфизмов есть дополнительные структуры $(n$-арные композиции и дифференциалы), и композиции обычных морфизмов ассоциативны только с точностью до цепной гомотопии. Такая структура назьвается $A_{\infty}$-категорией. Категория Фукая зависит только от расширенной симплектической структуры на $X$. Объекты категории Фукая - это, грубо говоря, тройки $(Y, E, \nabla)$, где $Y$ - лагранжево подмногообразие $X, E$ - комплексное расслоение на $Y$ с эрмитовой метрикой, а $\nabla$ - плоская унитарная связность на $E$. Это определение верно только "в первом приближении" по нескольким причинам. Во-первых, не каждое лагранжево подмногообразие $Y$ допустимо: так называемый класс Маслова $Y$ должен быть равен нулю. (Класс Маслова принимает значения в $H^{1}(Y, \mathbb{Z})$.) Во-вторых, $Y$ должно быть "градуировано". (Понятие градуированного лагранжева подмногообразия в симплектическом векторном пространстве было введено Ж. Лере в 1968 г. и обобщено М. Концевичем на случай лагранжевых подмногообразий в многообразиях Калаби-Яу.) В-третьих, возможно, требование унитарности связности $\nabla$ надо ослабить и потребовать только, чтобы собственные значения представления монодромии были по модулю равны 1. В-четвертых, если В-поле не равно нулю, то связность $\nabla$ должна быть только проективно плоской [19].

Морфизмы в категории Фукая определяются через так называемый комплекс Флоера. Это понятие будет обсуждаться в лекции 2.

Связь между гипотезой ГЗС, с одной стороны, и $A$ - и $B$-моделями, с другой, может быть объяснена следуюшим образом [25]. Если у нас имеется триангулированная (на самом деле, “оснащенная триангулированная" [3]) категория, то можно рассмотреть ее деформации. Информация о деформациях категории содержится в ее когомологиях Хохшильда. В случае $\mathbf{D}^{b}(X)$ когомологии Хохшильда, по всей видимости, изоморфны когомологиям внешней алгебры голоморфного касательного расслоения на $X$, т.е. пространству состояний $B$-модели. ${ }^{2}$ (См. также [42].) М. Концевич высказал гипотезу, что когомологии Хохшильда производной категории Фукая изоморфны квантовым когомологиям $X$, т.е. пространству состояний $A$-модели [25]. Если это так, то эквивалентность $\mathbf{D}^{b}(X)$ и $\mathbf{D} \mathscr{F}_{0}\left(X^{\prime}\right)$ влечет изоморфизм между $B$-моделью для $X$ и $A$-моделью для $X^{\prime}$, т.е. гомологическая зеркальная симметрия влечет слабую зеркальную симметрию.

Гипотеза ГЗС имеет интересный физический смысл. С точки зрения физики, основная идея состоит в том, чтобы обобщить понятие двумерной ТТП, разрешив мировому листу иметь границы (см., например, работу [47]). Это обобщение имеет смысл и для CKTП и приводит к понятию $D$-браны, которое играет очень важную роль в теории струн [37]. D-брана - это “хорошее” граничное условие для двумерной суперконформной теории поля. В квантовом случае не совсем ясно, какие граничные условия следует считать "хорошими", поэтому мы обсудим это понятие в рамках классической физики. Классическая двумерная теория поля задается своим функционалом действия, которьй является интегралом локального лагранжиана по мировому листу. До сих пор мы считали, что мировой лист имеет топологию цилиндра, образуюшие которого параметризованы временной координатой. Таким образом, одномерное пространство имело топологию окружности. Пусть теперь пространство имеет топологию отрез-

\footnotetext{
${ }^{2}$ Мы говорим "по всей видимости", посколшку до сих пор нет полного доказательства.
} 
ка $I$, т.е. мировой лист вьглядит как $\mathbb{R} \times I$. Чтобы классическая теория поля имела смысл, необходимо, чтобы задача Коши для уравнений Эйлера-Лагранжа имела однозначное решение, по крайней мере локально. Ясно, что для этого на поля и их производные надо наложить подходяшие условия на границах интервала. Например, можно потребовать зануления на границе (“условия Дирихле”) каких-нибудь скалярных полей, входящих в лагранжиан. ${ }^{3} D$-брана в классической теории поля - это просто выбор такого граничного условия.

Если теория поля имеет какие-нибудь симметрии, то можно потребовать, чтобы граничное условие сохраняло эти симметрии. Например, алгебра симметрий любой $N=1$ сигма-модели содержит прямую сумму двух копий $N=1$ супералгебры Вирасоро. Не сушествует граничных условий, которые сохраняли бы всю эту симметрию, но есть много граничных условий, которые сохраняют диагональную подалгебру. Такие граничные условия соответствуют обычным $D$-бранам в теории суперструн [37]. В случае $N=2$ мы имеем две копии $N=2$ супералгебры Вирасоро и можно потребовать, чтобы граничные условия сохраняли диагональную $N=2$ подалгебру. Такие граничные условия называются $D$-бранами типа $B$ или просто $B$-бранами, потому что они связаны с $B$-моделью (см. ниже). С другой стороны, можно использовать зеркальньй автоморфизм $\mathfrak{M}$ супералгебры Вирасоро и потребовать, чтобы граничное условие сохраняло другую $N=2$ подалгебру, натянутую на элементы

$$
L_{n}+\bar{L}_{n}, \quad-J_{n}+\bar{J}_{n}, \quad Q_{n}^{-}+\bar{Q}_{n}^{+}, \quad Q_{n}^{+}+\bar{Q}_{n}^{-}, \quad n \in \mathbb{Z} .
$$

Соответствуюшие $D$-браны назьваются $D$-бранами типа $A$ или просто $A$-бранами.

Если у нас есть классическая $D$-брана (типа $A$ или $B$ ), можно поставить задачу квантования классической теории поля на $\mathbb{R} \times I$ с граничными условиями, соответствующими этой $D$-бране. При этом мы требуем, чтобы квантование сохраняло $N=2$ супералгебру Вирасоро. Если это удается сделать, мы будем говорить, что классическая $D$-брана может быть проквантована, а классическая $D$-брана вместе с методом квантования будет назьваться квантовой $D$-браной. Полностью удовлетворительного квантового определения $D$-бран до сих пор не существует; найти такое определение - интересная задача на будущее.

Обсудим теперь связь между $A$ - и $B$-бранами и $A$ - и $B$-моделями. $A$ - и $B$-модели получаются из $N=2 \mathrm{CKTП} \mathrm{топологическим} \mathrm{скручиванием.} \mathrm{Грубо} \mathrm{говоря,} \mathrm{тополо-}$ гическое скручивание заключается в “усечении” теории до когомологии БРСТ-оператора $D_{A}$ или $D_{B}$. Заметим теперь, что оператор $D_{A}\left(\right.$ соответственно $\left.D_{B}\right)$ - элемент $N=2$ подалгебры Вирасоро, которая сохраняется граничньми условиями типа $A$ (соответственно типа $B$ ). Таким образом, граничные условия типа $A$ (соответственно $B$ ) согласованы со скручиванием типа $A$ (соответственно $B$ ). Иначе говоря, любая $A$-брана дает “хорошее" граничное условие для $A$-модели, а любая $B$-брана для $B$-модели. ${ }^{4}$

Можно показать, что множество $A$-бран (или $B$-бран) имеет структуру категории. Пространство морфизмов между двумя бранами $A$ и $A^{\prime}$ определяется как пространство состояний $2 d$-ТТП на $\mathbb{R} \times I$ с граничными условиями на концах интервала, соответствующими $A$ и $A^{\prime}$. Иначе говоря, надо рассмотреть пространство состояний

\footnotetext{
${ }^{3}$ Буква $D$ в слове " $D$-брана" происходит из слова "Dirichlet".

${ }^{4}$ Аксиомы двумерной ТТП с границами описаны в работах Г. Мура и Г. Сигала (неопубликовано; см. также [31]) и К. Лазароиу [27].
} 
$N=2$ СКТП на интервале и вычислить его когомологии по отношению к БРСТ-оператору $D_{A}$. Композиция морфизмов определяется через операторно-векторное соответствие $Y$ (точнее говоря, через его аналог для СКТП с границами).

Итак, любому физическому многообразию Калаби-Яу можно сопоставить две категории: категорию $A$-бран и категорию $B$-бран. Можно показать, что категория $A$-бран (соответственно $B$-бран) не зависит от "расширенных комплексных модулей" (соответственно "расширенных симплектических модулей") [47]. Удобно думать про эти категории как "обогащенные" $A$ - и $B$-модели: в то время как обычная $A$-модель это $2 d$-ТТП на мировом листе без границы, категория $A$-бран описьвает ту же самую ТТП на мировом листе со всевозможными граничными условиями. Ясно, что если два многообразия Калаби-Яу зеркальны друг другу, то категория $A$-бран для первого многообразия будет эквивалентна категории $B$-бран для второго, и наоборот. Ясно также, что если $N=2$ CКТП, соответствуюшие двум многообразиям Калаби-Яу, изоморфны, то соответствуюшие категории $A$-бран (или $B$-бран) эквивалентны.

Таким образом, чтобы доказать гипотезу ГЗС, достаточно доказать, что категория $A$-бран эквивалентна $\mathbf{D} \mathscr{F}_{0}(X)$, а категория $B$-бран эквивалентна $\mathbf{D}^{b}(X) . \mathrm{K}$ сожалению, сделать этого мы не можем даже в принщипе, поскольку отсутствует "честное" определение понятия $D$-браны. Можно показать, однако, что голоморфные векторные расслоения дают примеры $B$-бран, а объекты категории Фукая - примеры $A$-бран [47]. Виттен также показал, что в этих случаях морфизмы в категориях $A$-бран согласуются с морфизмами в категориях $\mathbf{D}^{b}(X)$ и $\mathbf{D} \mathscr{F}_{0}(X)$ соответственно. Это наблюдение Виттена и послужило мотивировкой гипотезы ГЗС. Позднее было показано, что более общие когерентные пучки, а также их комплексы тоже являются примерами $B$-бран. С другой стороны, в работе [20] было показано, что, вообще говоря, производная категория Фукая - это только подкатегория категории $A$-бран, т.е. для некоторых $X$ есть примеры $A$-бран, которые не изоморфны никакому объекту $\mathbf{D} \mathscr{F}_{0}(X)$. Это означает, что “симплектическая" сторона гипотезы ГЗС должна быть модифиширована. Мы обсудим это более подробно в лекции 4.

С математической точки зрения, гипотеза ГЗС не является четко сформулированным утверждением, поскольку проквантовать сигма-модель для произвольного многообразия Калаби-Яу не удается. Однако существует один класс многообразий Калаби-Яу, для которых квантование сигма-модели может быть проведено до конща и соответствующая $N=2$ CКТП может быть полностью описана: комплексные торы с плоской кэлеровой метрикой и произвольным $B$-полем. В случае торов можно дать точный критерий зеркальности в терминах линейной алгебры; это было сделано в работе [19] и будет описано в деталях в лекции 2 . Таким образом, в случае торов гипотеза ГЗС хорошо определена (за исключением вопросов, связанных с точньм определением категории Фукая). А. Полишук и Э. Заслоу доказали гипотезу ГЗС для торов вешественной размерности два [39]. Что касается торов большей размерности, то похоже, что для них ГЗС может быть верна только при условии существенной модификации определения категории Фукая. А именно, в категорию Фукая надо добавить много новых объектов. Это будет обсуждаться в лекщии 4.

Для всех остальных многообразий Калаби-Яу можно рассматривать гипотезу ГЗС как попытку математического определения отношения зеркальности. Тогда основная проблема состоит в том, чтобы дать строгое определение категории Фукая и доказать, что многочисленные примеры зеркальных многообразий, предложенные физиками и математиками, действительно зеркальны в смысле ГЗС. 
Другой подход к зеркальной симметрии был предложен в работе [41] и носит название SYZ-гипотезы. Согласно этой гипотезе, многообразия Калаби-Яу, входящие в зеркальные пары, обладают фибрациями, слои которых - специальные лагранжевы торы, дуальные друг другу в некотором смысле. Связь между гипотезами ГЗС и SYZ была исследована в работе [26].

\section{2. Зеркальная симметрия для плоских комплексных торов}

В этой лекции мы опишем $N=2$ суперконформные теории поля (СКТП), происходяшие из комплексных торов $T$ с плоской кэлеровой метрикой $G$ и постоянной 2 -формой $B$ (В-полем). Мы также дадим критерии того, когда два набора $(T, G, B)$ и $\left(T^{\prime}, G^{\prime}, B^{\prime}\right)$ производят изоморфные $N=2$ СКТП и когда их $N=2$ СКТП зеркально симметричны.

В лекции 1 было показано, что для определения $N=2$ суперконформной теории поля необходимо задать бесконечномерное $\mathbb{Z}_{2}$-градуированое векторное пространство состояний $V$, вакуумный вектор $|v a c\rangle$, операторно-векторное соответствие $Y$ из $V$ в пространство “формальных дробных степенных рядов от $z, \bar{z}$ с коэффициентами в $\operatorname{End}(V)$ ” и, наконец, элементы супералгебры Вирасоро $L, \bar{L}, Q^{ \pm}, \bar{Q}^{ \pm}, J, \bar{J}$, которые входят в определение суперконформной структуры (см. лекцию 1$)$.

Введем некоторые обозначения. Пусть $\Gamma \cong \mathbb{Z}^{2 d}$ - решетка в вещественном векторном пространстве $U$ размерности $2 d$ и $\Gamma^{*} \subset U^{*}$ - двойственная решетка. Рассмотрим вешественные торы $T=U / \Gamma, T^{*}=U^{*} / \Gamma^{*}$. Пусть $G$-метрика на $U$, т.е. положительно определенная симметрическая билинейная форма на $U$, а $B$ - вешественная кососимметричная форма на $U$. Обозначим через $l$ естественное спаривание $\Gamma \times \Gamma^{*} \rightarrow \mathbb{Z}$. (Естественное спаривание $U \times U^{*} \rightarrow \mathbb{R}$ также будем обозначать через l.) Выберем базис $e_{1}, \ldots, e_{2 d} \in \Gamma$. Компоненты элемента $w \in \Gamma$ в данном базисе будем обозначать через $w^{i}, i=1, \ldots, 2 d$. Компоненты элемента $m \in \Gamma^{*}$ в двойственном базисе обозначим через $m_{i}, i=1, \ldots, 2 d$. Через $G_{i j}, B_{i j}$ обозначим компоненты $G$ и $B$ в том же самом базисе. Далее будет видно, что суперконформная теория поля, которую мы построим, не будет зависеть от выбора базиса в $\Gamma$. В физической литературе $\Gamma$ называется решеткой намоток, а $\Gamma^{*}$ - решеткой моментов.

Рассмотрим тройку $(T, G, B)$. Каждой такой тройке мы сопоставим $N=2$ суперконформную теорию поля $\operatorname{SCFT}(\Gamma, I, G, B)$, которую можно рассматривать как квантование суперсимметричной сигма-модели.

Пространство состояний СКТП $\mathscr{V}$ - это тензорное произведение

$$
V=\mathscr{H}_{b} \otimes_{\mathbb{C}} \mathscr{H}_{f} \otimes_{\mathbb{C}} \mathbb{C}\left[\Gamma \oplus \Gamma^{*}\right]
$$

в котором $\mathscr{H}_{b}$ и $\mathscr{H}_{f}-$ бозонное и фермионное пространства Фока, определяемые ниже, в то время как $\mathbb{C}\left[\Gamma \oplus \Gamma^{*}\right]-$ пространство групповой алгебры решетки $\Gamma \oplus \Gamma^{*}$ над $\mathbb{C}$.

Для определения пространства $\mathscr{H}_{b}$ рассмотрим алгебру над $\mathbb{C}$ с образуюшими $\alpha_{s}^{i}$, $\bar{\alpha}_{s}^{i}, i=1, \ldots, 2 d, s \in \mathbb{Z} \backslash 0$, и соотношениями

$$
\left[\alpha_{s}^{i}, \alpha_{p}^{j}\right]=s\left(G^{-1}\right)^{i j} \delta_{s,-p}, \quad\left[\bar{\alpha}_{s}^{i}, \bar{\alpha}_{p}^{j}\right]=s\left(G^{-1}\right)^{i j} \delta_{s,-p}, \quad\left[\alpha_{s}^{i}, \bar{\alpha}_{p}^{j}\right]=0 .
$$

Для положительного целого $s$ элементы $\alpha_{-s}^{i}$ и $\bar{\alpha}_{-s}^{i}$ называются соответственно левыми и правьми бозонными операторами рождения, в противоположном случае они 
называются левыми и правыми бозонньми операторами уничтожения (аннигиляторами). Операторы рождения и уничтожения вместе назьваются осцилляторами.

Пространство $\mathscr{H}_{b}$ определяется как пространство полиномов от четных переменных $a_{-s}^{i}, \bar{a}_{-s}^{i}, i=1, \ldots, 2 d, s=1,2, \ldots$ Бозонная осцилляторная алгебра действует на $\mathscr{H}_{b}$ по правилу

$$
\begin{aligned}
\alpha_{-s}^{i} & \mapsto a_{-s}^{i}, & \bar{\alpha}_{-s}^{i} & \mapsto \bar{a}_{-s}^{i}, \\
\alpha_{s}^{i} & \mapsto s\left(G^{-1}\right)^{i j} \frac{\partial}{\partial a_{-s}^{j}}, & \bar{\alpha}_{s}^{i} & \mapsto s\left(G^{-1}\right)^{i j} \frac{\partial}{\partial \bar{a}_{-s}^{j}}
\end{aligned}
$$

для всех положительных $s$. Это представление бозонной осцилляторной алгебры называется представлением Фока-Баргманна. Вектор $1 \in \mathscr{H}_{b}$ зануляется всеми бозонными аннигиляторами и будет обозначаться $\left|v a c_{b}\right\rangle$.

Пространство $\mathscr{H}_{b}$ рассматривается как $\mathbb{Z}_{2}$-градуированное векторное пространство с тривиальной (чисто четной) градуировкой. Очевидно, что $\mathscr{H}_{b}$ раскладывается в произведение $\mathfrak{H}_{b} \otimes \overline{\mathfrak{H}}_{b}$, где $\mathfrak{H}_{b}$ (соответственно $\overline{\mathfrak{H}}_{b}$ ) - бозонное пространство Фока, порожденное лишь левыми (соответственно правыми) бозонными осцилляторам.

Для определения $\mathscr{H}_{f}$ рассмотрим алгебру над $\mathbb{C}$ с порождаюшими $\psi_{s}^{i}, \bar{\psi}_{s}^{i}, i=$ $1, \ldots, 2 d, s \in \mathbb{Z}+\frac{1}{2}$, и соотношениями

$$
\left\{\psi_{s}^{i}, \psi_{p}^{j}\right\}=\left(G^{-1}\right)^{i j} \delta_{s,-p}, \quad\left\{\bar{\psi}_{s}^{i}, \bar{\psi}_{p}^{j}\right\}=\left(G^{-1}\right)^{i j} \delta_{s,-p}, \quad\left\{\psi_{s}^{i}, \bar{\psi}_{p}^{j}\right\}=0 .
$$

Когда $s$ положительно, $\psi_{-s}^{i}$ и $\bar{\psi}_{-s}^{i}$ называются левыми и правыми фермионньми операторами рождения, в противном случае они называются левыми и правыми фермионными аннигиляторами. Вместе они назьваются фермионньми осцилляторами.

Пусть $\mathscr{H}_{f}$ будет пространством косых полиномов от нечетных переменных $\theta_{-s}^{i}, \bar{\theta}_{-s}^{i}$, $i=1, \ldots, 2 d, s=1 / 2,3 / 2, \ldots$ Фермионная осцилляторная алгебра действует на $\mathscr{H}_{f}$ по правилу

$$
\begin{array}{rlrl}
\psi_{-s}^{i} & \mapsto \theta_{-s}^{i}, & \bar{\psi}_{-s}^{i} \mapsto \bar{\theta}_{-s}^{i}, \\
\psi_{s}^{i} \mapsto\left(G^{-1}\right)^{i j} \frac{\partial}{\partial \theta_{-s}^{j}}, & \bar{\psi}_{s}^{i} \mapsto\left(G^{-1}\right)^{i j} \frac{\partial}{\partial \bar{\theta}_{-s}^{j}}
\end{array}
$$

для всех положительных $s \in \mathbb{Z}+\frac{1}{2}$. Это есть представление Фока-Баргманна для фермионной осцилляторной алгебры. Вектор $1 \in \mathscr{H}_{f}$ зануляется всеми фермионными аннигиляторами и обозначается $\left|v a c_{f}\right\rangle$. Фермионное пространство Фока имеет естественную $\mathbb{Z}_{2}$-градуировку, в которой вектор $\left|v a c_{f}\right\rangle$ является четным. Существует разложение $\mathfrak{H}_{f} \otimes \overline{\mathfrak{H}}_{f}$, где $\mathfrak{H}_{f}$ (соответственно $\overline{\mathfrak{H}}_{f}$ ) строятся с использованием только левых (соответственно правых) фермионных осцилляторов.

Для $w \in \Gamma, m \in \Gamma^{*}$ обозначим вектор $w \oplus m \in \mathbb{C}\left[\Gamma \oplus \Gamma^{*}\right]$ через $(w, m)$. Будем также использовать обозначение $|v a c, w, m\rangle$ вместо

$$
\left|v a c_{b}\right\rangle \otimes\left|v a c_{f}\right\rangle \otimes(w, m) .
$$


Определение $\mathscr{V}$ требует указать вакуумньй вектор, элементы $T, \bar{T}$ и операторно-векторное соответствие $Y$. Для этого введем вспомогательные объекты. Определим операторы $W: V \rightarrow V \otimes \Gamma$ и $M: V \rightarrow V \otimes \Gamma^{*}$ по правилу

$W^{i}: b \otimes f \otimes(w, m) \mapsto w^{i}(b \otimes f \otimes(w, m)), \quad M_{i}: b \otimes f \otimes(w, m) \mapsto m_{i}(b \otimes f \otimes(w, m))$.

Также положим

$$
\begin{gathered}
\partial X^{j}(z)=\frac{1}{z}\left(G^{-1}\right)^{j k} P_{k}+\sum_{s=-\infty}^{\infty} \frac{\alpha_{s}^{j}}{z^{s+1}}, \quad \bar{\partial} X^{j}(\bar{z})=\frac{1}{\bar{z}}\left(G^{-1}\right)^{j k} \bar{P}_{k}+\sum_{s=-\infty}^{\infty} \frac{\bar{\alpha}_{s}^{j}}{\bar{z}^{s+1}}, \\
\psi^{j}(z)=\sum_{r \in \mathbb{Z}+\frac{1}{2}} \frac{\psi_{r}^{j}}{z^{r+1 / 2}}, \quad \bar{\psi}^{j}(\bar{z})=\sum_{r \in \mathbb{Z}+\frac{1}{2}} \frac{\bar{\psi}_{r}^{j}}{\bar{z}^{r+1 / 2}},
\end{gathered}
$$

где штрих над суммой означает, что сумма берется по всем $s$ за исключением $s=0$, а $P_{k}$ и $\bar{P}_{k}$ задаются формулами

$$
P_{k}=\frac{1}{\sqrt{2}}\left(M_{k}+\left(-B_{k j}-G_{k j}\right) W^{j}\right), \quad \bar{P}_{k}=\frac{1}{\sqrt{2}}\left(M_{k}+\left(-B_{k j}+G_{k j}\right) W^{j}\right) .
$$

Мы не определяем сами $X^{j}(z, \bar{z})$, а только их производные. Причина состоит в том, что поля $X^{j}(z, \bar{z})$ содержат члены, пропорциональные $\log z$ и $\log \bar{z}$, и, таким образом, не являются "дробными степенными рядами".

Вакуумный вектор в $\mathscr{V}$ определяется по правилу

$$
|v a c\rangle=|v a c, 0,0\rangle \text {. }
$$

Общая формула для операторно-векторного соответствия $Y$ достаточно сложна и может быть найдена в [19]. Мы приведем только формулы для некоторых частных случаев. Векторы $\alpha_{-s}^{j}|v a c, 0,0\rangle$ и $\bar{\alpha}_{-s}^{j}|v a c, 0,0\rangle$ под действием $Y$ переходят в

$$
\frac{1}{(s-1) !} \partial^{s} X^{j}(z), \quad \frac{1}{(s-1) !} \bar{\partial}^{s} X^{j}(\bar{z}) \text {. }
$$

Векторы $\psi_{-s}^{j}|v a c, 0,0\rangle$ и $\bar{\psi}_{-s}^{j}|v a c, 0,0\rangle$ отображаются в

$$
\frac{1}{\left(s-\frac{1}{2}\right) !} \partial^{s-1 / 2} \psi^{j}(z), \quad \frac{1}{\left(s-\frac{1}{2}\right) !} \bar{\partial}^{s-1 / 2} \bar{\psi}^{j}(\bar{z}) .
$$

Для задания $N=2$ суперконформной структуры на $\mathscr{V}$ достаточно выбрать комплексную структуру $I$ на $U$, относительно которой метрика $G$ является кэлеровой. Обозначим через $\omega=G I$ соответствующую кэлерову форму. Тогда левобегушие векторы определяются по следуюшим формулам:

$$
\begin{aligned}
L & =\frac{1}{2} G\left(a_{-1}, a_{-1}\right)-\frac{1}{2} G\left(\theta_{-1 / 2}, \theta_{-3 / 2}\right), \\
Q^{ \pm} & =\frac{-i}{4 \sqrt{2}}(G \mp i \omega)\left(\theta_{-1 / 2}, a_{-1}\right), \\
J & =-\frac{i}{2} \omega\left(\theta_{-1 / 2}, \theta_{-1 / 2}\right) .
\end{aligned}
$$


Правобегущие векторы $\bar{L}, \bar{Q}^{ \pm}$и $\bar{J}$ определяются по тем же правилам с заменой $a$ на $\bar{a}$ и $\theta$ на $\bar{\theta}$.

Изоморфизм $N=2$ СКТП - это изоморфизм подлежаших векторных пространств $f: V \stackrel{\sim}{\rightarrow} V^{\prime}$, сохраняюший операторно-векторное соответствие

$$
Y^{\prime}(f(a)) f(b)=f(Y(a) b)
$$

и действующий тождественно на образующих как правой, так и левой $N=2$ супералгебры Вирасоро.

Зеркальныи й морфизм между двумя $N=2$ CКТП - это изоморфизм между подле-

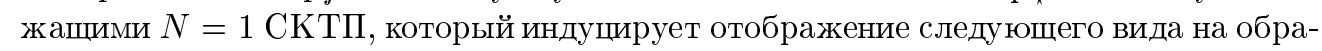
зуюших левой/правой супералгебр Вирасоро:

$$
\begin{aligned}
& f(L)=L^{\prime}, \quad f\left(Q^{+}\right)=Q^{-^{\prime}}, \quad f\left(Q^{-}\right)=Q^{+^{\prime}}, \quad f(J)=-J^{\prime}, \\
& f(\bar{L})=\bar{L}^{\prime}, \quad f\left(\bar{Q}^{+}\right)=\bar{Q}^{+^{\prime}}, \quad f\left(\bar{Q}^{-}\right)=\bar{Q}^{-^{\prime}}, f(\bar{J})=\bar{J}^{\prime} .
\end{aligned}
$$

Композиция двух зеркальных морфизмов является изоморфизмом $N=2$ СКТП.

Теперь мы готовы дать описание того, когда две четверки $(\Gamma, I, G, B)$ и $\left(\Gamma^{\prime}, I^{\prime}, G^{\prime}, B^{\prime}\right)$ определяют изоморфные $N=2$ СКТП и когда они зеркально симметричны.

Естественное спаривание $l: \Gamma \oplus \Gamma^{*} \rightarrow \mathbb{Z}$ индуцирует $\mathbb{Z}$-значную симметричную билинейную форму $q$ на $\Gamma \oplus \Gamma^{*}$, задаваемую формулой

$$
q\left(\left(w_{1}, m_{1}\right),\left(w_{2}, m_{2}\right)\right)=l\left(w_{1}, m_{2}\right)+l\left(w_{2}, m_{1}\right), \quad w_{1,2} \in \Gamma, \quad m_{1,2} \in \Gamma^{*} .
$$

Имея $G, I, B$, можно определить две комплексные структуры на $T \times T^{*}$ :

$$
\begin{aligned}
\mathscr{I}(I, B) & =\left(\begin{array}{cc}
I & 0 \\
B I+I^{t} B & -I^{t}
\end{array}\right), \\
\mathscr{J}(G, I, B) & =\left(\begin{array}{cc}
-I G^{-1} B & I G^{-1} \\
G I-B I G^{-1} B & B I G^{-1}
\end{array}\right) .
\end{aligned}
$$

Здесь используются следуюшие обозначения. Мы рассматриваем $\mathscr{I}$ и $\mathscr{J}$ как эндоморфизмы $U \oplus U^{*}$ и записьваем их матрицы в базисе, в котором первые $2 d$ вектора порождают $U$, а оставшиеся порождают $U^{*}$. Кроме того, $G$ и $B$ рассматриваются как элементы $\operatorname{Hom}\left(U, U^{*}\right)$, а $I^{t}$ - это эндоморфизм $U^{*}$, сопряженный к $I$.

Сразу видно, что $\mathscr{J}$ зависит от $G, I$ только в комбинации $\omega=G I$, т.е. зависит только от симплектической структуры на $T$ и В-поля. Сушествует также и третья естественная комплексная структура $\widetilde{\mathscr{I}}$ на $T \times T^{*}$, которая есть просто комплексная структура произведения $T \times T^{*}$ двух комплексных многообразий:

$$
\tilde{\mathscr{I}}=\left(\begin{array}{cc}
I & 0 \\
0 & -I^{t}
\end{array}\right) .
$$

Эта комплексная структура не будет играть значительной роли в дальнейшем. Заметим только, что $\mathscr{I}$ совпадает с $\widetilde{\mathscr{I}}$, если и только если $B^{(0,2)}=0$.

Teopema 2.1 [19]. $\operatorname{SCFT}(\Gamma, I, G, B)$ изоморфна $\operatorname{SCFT}\left(\Gamma^{\prime}, I^{\prime}, G^{\prime}, B^{\prime}\right)$ тогда и только тогда, когда существует изоморфизм решеток $\Gamma \oplus \Gamma^{*}$ u $\Gamma^{\prime} \oplus \Gamma^{\prime *}$, который переводит q в $q^{\prime}, \mathscr{I}$ в $\mathscr{I}^{\prime}$ и $\mathscr{J}$ в $\mathscr{J}^{\prime}$.

Вторая теорема дает критерий зеркальной симметрии $(T, I, G, B)$ и $\left(T^{\prime}, I^{\prime}, G^{\prime}, B^{\prime}\right)$. 
Teорема 2.2 [19]. $\operatorname{SCFT}(\Gamma, I, G, B)$ зеркально симметрична $\operatorname{SCFT}\left(\Gamma^{\prime}, I^{\prime}, G^{\prime}, B^{\prime}\right)$ тогда и только тогда, когда существует изоморфизм решеток $\Gamma \oplus \Gamma^{*} u \Gamma^{\prime} \oplus \Gamma^{\prime}$, который переводит q в $q^{\prime}, \mathscr{I}$ в $\mathscr{J}^{\prime}$ и $\mathscr{J}$ в $\mathscr{I}^{\prime}$.

Данная теорема позволяет строить большое количество примеров зеркально симметричных торов. Предположим, что задан комплексный тор $(T, I)$ с постоянной кэлеровой формой $\omega$, и предположим, что $T=A \times B$, где $A$ и $B$ - лагранжевы относительно $\omega$ подторы. В частности, решетка Г представляется в виде $\Gamma_{A} \oplus \Gamma_{B}$. Пусть $\widehat{A}$ - тор, двойственный к $A$, и пусть $T^{\prime}=\widehat{A} \times B$. Решетка, соответствующая $T^{\prime},-$ это $\Gamma^{\prime}=\Gamma_{A}^{*} \oplus \Gamma_{B}$. Сушествует очевидный изоморфизм между $\Gamma \oplus \Gamma^{*}$ и $\Gamma^{\prime} \oplus \Gamma^{\prime *}$, который переводит $q$ в $q^{\prime}$. Обозначив через $\mathscr{I}^{\prime}$ и $\mathscr{J}^{\prime}$ образы $\mathscr{J}$ и $\mathscr{I}$ соответственно и обратив соотношения между $\mathscr{I}^{\prime}, \mathscr{J}^{\prime}$ и $I^{\prime}, \omega^{\prime}, B^{\prime}$, можно получить комплексную структуру, симплектическую форму и В-поле на $T^{\prime}$. Нетрудно проверить, что данная процедура дает комплексньй тор с плоской кэлеровой метрикой и В-полем, которьй зеркально симметричен изначальному тору. Данный процедура является частньм случаем такого физического понятия, как Т-двойственность [36].

Вскоре мы покажем, как данные результаты могут быть использованы при тестировании гипотезы о гомологической зеркальной симметрии для плоских торов. Вначале напомним формулировку данной гипотезы.

Физическое многообразие Калаби-Яу $(X, G, B)$ - это одновременно комплексное и симплектическое многообразие (симплектической формой является кэлерова форма $\omega=G I$ ). Каждому такому многообразию можно сопоставить пару триангулированных категорий: ограниченную производную категорию когерентных пучков $\mathbf{D}^{b}(X)$ и производную категорию Фукая $\mathbf{D} \mathscr{F}_{0}(X)$. Первая зависит только от комплексной структуры на $X$, в то время как вторая зависит только от симплектической формы. Гипотеза о гомологической зеркальной симметрии (ГЗС) утверждает, что если два алгебраических многообразий Калаби-Яу $(X, G, B)$ и $\left(X^{\prime}, G^{\prime}, B^{\prime}\right)$ зеркально симметричны, то $\mathbf{D}^{b}(X)$ эквивалентна $\mathbf{D} \mathscr{F}_{0}\left(X^{\prime}\right)$, и наоборот, $\mathbf{D}^{b}\left(X^{\prime}\right)$ эквивалентна $\mathbf{D} \mathscr{F}_{0}(X)$.

Теперь мы должны напомнить определения этих двух категорий. Мы начнем с категории Фукая $\mathscr{F}(X)$. Объектами данной категории являются тройки $\left(Y, E, \nabla_{E}\right)$, в которых $Y$ - это градуированное лагранжево подмногообразие $X, E$ - комплексное векторное расслоение на $Y$ с эрмитовой метрикой, $\nabla_{E}-$ плоская унитарная связность на $E$. Понятие, которое требует пояснений, - это "градуированное лагранжево подмногообразие". Оно было введено Лере в 1968 г. для лагранжевых подмногообразий симплектического аффинного пространства и было обобщено М. Конщевичем на случай многообразия Калаби-Яу [25]. Вначале напомним определение класса Маслова для лагранжева подмногообразия. Выберем голоморфное сечение $\Omega$ канонического линейного расслоения (которое тривиально для многообразий Калаби-Яу). Ограничивая его на $Y$, получим $n$-форму, нигде не обрашающуюся в нуль. С другой стороны, на $Y$ имеется форма объема vol, индуцированная кэлеровой метрикой на $X$. Это также нигде не обрашаюшаяся в нуль $n$-форма на $Y$, и, значит, $\left.\Omega\right|_{Y}=h \cdot v o l$, где $h$ - нигде не обрашающаяся в нуль комплексная функция на $Y$. Функцию $h$ можно рассматривать как элемент $H^{0}\left(Y, \mathscr{C}_{Y}^{\infty}\right)$, где $\mathscr{C}_{Y}^{\infty} *$ - пучок гладких $\mathbb{C}^{*}$-значных функций на $Y$. Стандартная экспоненциальная точная последовательность задает гомоморфизм из $H^{0}\left(Y, \mathscr{C}_{Y}^{\infty *}\right)$ в $H^{1}(Y, \mathbb{Z})$, и класс Маслова у $Y$ определяется как образ $h$ относительно этого гомоморфизма. (Явно коцикл Чеха, представляюший класс Маслова, строит- 
ся так: выбирается хорошее покрытие $Y$, берется логарифм $h$ для каждого элемента покрытия, делится на $2 \pi i$, и результаты сравниваются на пересечениях.) Несмотря на то, что определение класса Маслова на первьй взгляд зависит и от комплексной, и от симплектической структур на $X$, в действительности, он не должен зависеть от выбора комплексной структуры. Отметим, что если класс Маслова зануляется, то логарифм $h$ определен как функция и единственен с точностью до $2 \pi i m, m \in \mathbb{Z}$. Градуированное лагранжево подмногообразие $Y$ - это лагранжево подмногообразие в $X$ с нулевым классом Маслова и выбором ветви $\log h$.

Морфизмы определяются таким образом. Пусть заданы два объекта $\left(Y_{1}, E_{1}, \nabla_{1}\right)$ и $\left(Y_{2}, E_{2}, \nabla_{2}\right)$. Будем предполагать, что $Y_{1}$ и $Y_{2}$ пересекаются трансверсально в конечном числе точек; если это не так, то продеформируем один из объектов вдоль гамильтонова векторного поля, пока не добьемся трансверсальности. Пусть $\left\{e_{i}, i \in I\right\}$ - множество точек пересечения $Y_{1}$ и $Y_{2}$. Рассмотрим комплекс пространство это прямая сумма

$$
V_{i}=\operatorname{Hom}\left(E_{1}\left(e_{i}\right), E_{2}\left(e_{i}\right)\right), \quad i \in I .
$$

Градуировка определяется по следующему правилу. $\mathrm{B}$ каждой точке $p \in Y$ пространство $T_{p} Y$ задает точку $q$ в грассманиане лагранжевых подпространств в $T_{p} X$. Обозначим через $\widetilde{L a g_{p}}$ универсальные накрытия лагранжевых грассманианов пространств $T_{p} X$. На многообразиях Калаби-Яу $X$ эти накрытия склеиваются в слоение, обозначаемое $\widetilde{L a g}[25]$. Градуировка $Y$ задает канонический подъем $q$ в $\widetilde{L a g}_{p}$ для всех $p$; эти подъемы склеиваются в сечение для ограничения $\widetilde{L a g}$ на $Y$ [25]. Таким образом, для каждой точки пересечения $e_{i}$ получается пара точек $q_{1}, q_{2} \in \widetilde{L a g}_{e_{i}}$. Градуировка компоненты комплекса Флоера, соответствующей $e_{i}$, - это индекс Маслова пары $q_{1}, q_{2}$ (см. [4] для определения индекса Маслова). В заключение определим дифференциал. Пусть $e_{i}$ и $e_{j}$ - пара точек, градуировки которых отличаются на единицу. Компонента дифференциала Флоера, которая действует из $V_{i}$ в $V_{j}$, определяется подсчетом голоморфных дисков в $X$ с двумя отмеченньми точками на гранище, совпадаюшими с $e_{i}$ и $e_{j}$ (индекс Маслова $e_{j}$ больше на единищу индекса Маслова $e_{i}$ ) и такими, у которых две части границы попадают одна на $Y_{1}$, а другая на $Y_{2}$. Отметим, что для вычисления дифференциала нужна (почти) комплексная структура $J$ на $X$, для которой $\omega(\cdot, J \cdot)$ является эрмитовой формой на касательном расслоении к $X$. Точное определение дифференциала Флоера см. в [9]. Пространство морфизмов в категории Фукая - это комплекс Флоера. Для определения композиции используются голоморфные диски с тремя отмеченными точками и границей, лежащей на трех лагранжевых подмногообразиях.

Определение, набросанное вьше, является приблизительным. Во-первых, для определения дифференциала Флоера нужно зафиксировать относительную спин-структуру на $Y[9]$. Во-вторых, квадрат дифференциала не всегда равен 0, т.е. "комплекс" Флоера не всегда комплекс. Другая проблема состоит в том, что композиция морфизмов ассоциативна лишь с точностью до гомотопии, зависяшей от тернарного произведения. В действительности, сушествует бесконечная серия высших произведений в категории Фукая, которые предположительно формируют $A_{\infty}$-категорию (см. [24] в качестве обзора по $A_{\infty}$-категориям). Существует уверенность в том, что изменение почти комплексной структуры $J$ не меняет $A_{\infty}$-категорию, т.е. категория Фукая является симплектическим инвариантом. Подробную дискуссию можно майти в [9]. 
Для нетривиального В-поля категория Фукая должна быть модифицирована следуюшим образом. Объекты - это тройки $\left(Y, E, \nabla_{E}\right)$, где $Y$-градуированное лагранжево подмногообразие, $E$ - векторное расслоение на $Y$ с эрмитовой метрикой, а $\nabla_{E}-$ эрмитова связность на $E$ с кривизной

$$
\nabla_{E}^{2}=\left.2 \pi i B\right|_{Y}
$$

Таким образом, связность должна быть проективно плоской, а не плоской.

Морфизмы модифицируются так: симплектическая форма $\omega$ в определении комплекса Флоера и высших произведений во всех местах заменяется на $\omega+i B$. Модифицированная категория Фукая симплектического многообразия $X$ с В-полем $B$ будет обозначатся $\mathscr{F}(X, B)$.

Категория Фукая $\mathscr{F}(X, B)$ не является категорией в обычном смысле, а есть $A_{\infty}$-категория с функтором сдвига. Морфизмы между двумя объектами в $A_{\infty}$-категории это дифференциально градуированные векторные пространства. С каждой $A_{\infty}$-категорией связьвается обычная категория с теми же самыми объектами и 0 -й групой когомологий в качестве морфизмов. Применяя эту конструкцию к $\mathscr{F}(X, B)$, получим категорию $\mathscr{F}_{0}(X, B)$, которая также называется категорией Фукая. Рассматривая скрученные комплексы над $\mathscr{F}(X, B)$, М. Концевич [25] также определил некоторую триангулированную категорию $\mathbf{D} \mathscr{F}_{0}(X, B)$. Будем называть ее производной категорией Фукая. Категория $\mathbf{D} \mathscr{F}_{0}(X, B)$ содержит $\mathscr{F}_{0}(X, B)$ в качестве полной подкатегории.

В следующей лекции мы обсудим производные категории когерентных пучков и, используя их свойства, протестируем гипотезу о гомологической зеркальной симметрии.

\section{3. Производные категории когерентных пучков и тестирование гипотезы о гомологической зеркальной симметрии}

Пусть $X$ - алгебраическое многообразие над $\mathbb{C}$ (или комплексное многообразие). Обозначим через $\mathscr{O}_{X}$ пучок регулярных функций (или пучок голоморфных функций). Напомним, что когерентным пучком назьвается пучок $\mathscr{O}_{X}$-модулей, которьй локально может быть представлен как коядро морфизма алгебраических (голоморфных) векторных расслоений конечного ранга. Когерентные пучки образуют абелеву категорию, которая будет обозначатся $\operatorname{coh}(X)$.

Далее мы напомним определение производной категории и опишем некоторые свойства производных категорий когерентных пучков на гладких проективных многообразиях. Сушествует множество текстов, где может быть найдено введение в теорию производных и триангулированных категорий; мы рекомендуем [43], [15], [12], [21], [23].

Пусть $\mathscr{A}$ - абелева категория. Обозначим через $\mathbf{C}^{b}(\mathscr{A})$ категорию ограниченных дифференциальных комплексов

$$
M^{*}=\left(0 \longrightarrow \cdots \longrightarrow M^{p} \stackrel{d^{p}}{\longrightarrow} M^{p+1} \longrightarrow \cdots \longrightarrow 0\right), \quad M^{p} \in \mathscr{A}, \quad p \in \mathbb{Z}, \quad d^{2}=0 .
$$

Морфизмы $f: M^{\cdot} \longrightarrow N^{\cdot}$ между комплексами - это наборы морфизмов $f^{p}: M^{p} \longrightarrow$ $N^{p}$ в категории $\mathscr{A}$, которые коммутируют с дифференциалами, т.е.

$$
d_{N} f^{p}-f^{p+1} d_{M}=0 \text { для всех } p .
$$


Морфизм комплексов $f: M^{\cdot} \longrightarrow N^{\cdot}$ гомотопен нулю, если $f^{p}=d_{N} h^{p}+h^{p+1} d_{M}$ для всех $p \in \mathbb{Z}$ и для некоторого семейства морфизмов $h^{p}: M^{p+1} \longrightarrow N^{p}$. Определим гомотопическую категорию $\mathbf{H}^{b}(\mathscr{A})$ как категорию, которая имеет те же самые объекты что и $\mathbf{C}^{b}(\mathscr{A})$, в то время как морфизмы в $\mathbf{H}^{b}(\mathscr{A})$ - это классы эквивалентности морфизмов между комплексами $\bar{f}$, по модулю гомотопных нулю.

Для любого комплекса $N^{\cdot}$ и для каждого $p \in \mathbb{Z}$ определим когомологию $H^{p}\left(N^{*}\right) \in$ $\mathscr{A}$ как фактор $\operatorname{Ker} d^{p} / \operatorname{Im} d^{p-1}$. Таким образом, для каждого $p$ получаем функтор $H^{p}: \mathbf{C}(\mathscr{A}) \longrightarrow \mathscr{A}$, которьй сопоставляет комплексу $N^{\cdot}$ его когомологию $H^{p}\left(N^{*}\right) \in \mathscr{A}$. Определим квазиизоморфизм как такой морфизм комплексов $s: N^{\cdot} \rightarrow M^{\cdot}$, для которого индуцируемые морфизмы $H^{p} s: H^{p}\left(N^{*}\right) \rightarrow H^{p}\left(M^{*}\right)$ являются изоморфизмами для всех $p \in \mathbb{Z}$. Обозначим через $\Sigma$ класс всех квазиизоморфизмов. Этот класс морфизмов обладает хорошими свойствами, аналогичными условиям Оре в теории локализации колец.

Ограниченная производная категория $\mathbf{D}^{b}(\mathscr{A})$ определяется как локализация $\mathbf{H}^{b}(\mathscr{A})$ относительно класса всех квазиизоморфизмов $\Sigma$. Это означает, что производная категория имеет те же самые объекты, что и гомотопическая категория $\mathbf{H}^{b}(\mathscr{A})$, а морфизмы в ней из $N^{\cdot}$ в $M^{\cdot}$ задаются как левые частные $s^{-1} \circ f$, т.е. классами эквивалентности диаграмм

$$
N^{\cdot} \stackrel{f}{\longrightarrow} M^{\prime \cdot} \stackrel{s}{\longleftarrow} M^{\cdot}, \quad s \in \Sigma .
$$

Пары $(f, s)$ и $(g, t)$ считаются эквивалентными, если и только если сушествует коммутативная диаграмма в $\mathbf{H}^{b}(\mathscr{A})$

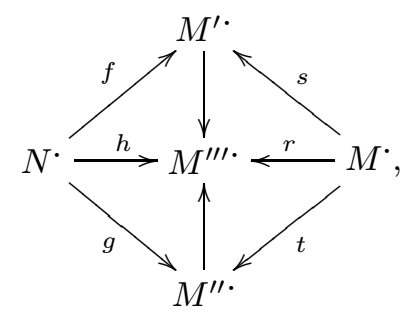

в которой $r \in \Sigma$. Композиция морфизмов $(f, s)$ и $(g, t)$ - это морфиим $\left(g^{\prime} f, s^{\prime} t\right)$, который определяется с использованием следующей диаграммы:

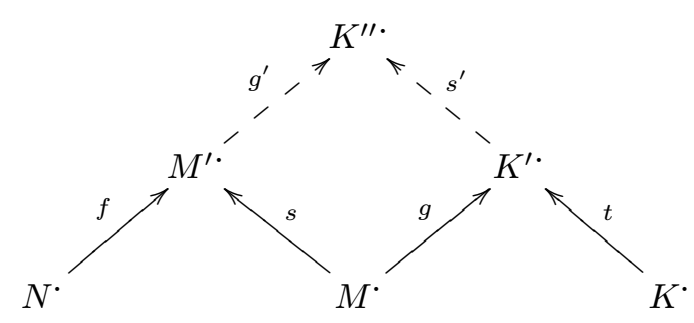

Такая диаграмма всегда существует, и можно проверить ассоциативность данного закона композиции.

Имеется канонический функтор $\mathbf{H}^{b}(\mathscr{A}) \longrightarrow \mathbf{D}^{b}(\mathscr{A})$, переводящий $f: N^{\cdot} \rightarrow M^{\cdot}$ в пару $\left(f, \mathrm{id}_{M}\right)$. Данньй функтор обрашает все квазиизоморфизмы и является универсальным среди функторов, обладающих этим свойством. Абелева категория $\mathscr{A}$ вкла- 
дьвается как полная подкатегория в $\mathbf{D}^{b}(\mathscr{A})$, отождествляя объект $A \in \mathscr{A}$ с комплексом $\cdots \rightarrow 0 \rightarrow A \rightarrow 0 \rightarrow \cdots$, имеюшим $A$ в степени 0 . Для произвольного комплекса $N^{\cdot}$ обозначим через $N^{\cdot}[1]$ комплекс с компонентами $N^{\cdot}[1]^{p}=N^{p+1}$ и диффференщиалом $d_{N[1]}=-d_{N}$. Данное соответствие определяет функтор на производной категории $\mathbf{D}^{b}(\mathscr{A})$, которьй является автоэквивалентностью и назьвается функтором сдвига.

Каждая производная категория $\mathbf{D}^{b}(\mathscr{A})$ имеет структуру триангулированной категории [43]. Это означает, что заданы следуюшие данные:

а) функтор сдвига (см. [1]) $\mathbf{D}^{b}(\mathscr{A}) \longrightarrow \mathbf{D}^{b}(\mathscr{A})$, являюшийся аддитивной автоэквивалентностью,

b) класс выделенных (или точных) треугольников

$$
X \stackrel{u}{\longrightarrow} Y \stackrel{v}{\longrightarrow} Z \stackrel{w}{\longrightarrow} X[1]
$$

и эти данные удовлетворяют определенному набору аксиом (см. [43], [12], [21], [23]).

Для задания триангулированной структуры на производной категории $\mathbf{D}^{b}(\mathscr{A})$ вводится понятие стандартного треугольника как последовательности

$$
N \stackrel{Q i}{\longrightarrow} M \stackrel{Q p}{\longrightarrow} K \stackrel{\partial \varepsilon}{\longrightarrow} N[1]
$$

где $Q: \mathbf{C}^{b}(\mathscr{A}) \longrightarrow \mathbf{D}^{b}(\mathscr{A})$ - канонический функтор,

$$
0 \longrightarrow N \stackrel{i}{\longrightarrow} M \stackrel{p}{\longrightarrow} K \longrightarrow 0
$$

- короткая точная последовательность комплексов и $\partial \varepsilon-$ некоторьй морфизм в $\mathbf{D}^{b}(\mathscr{A})$. Морфизм $\partial \varepsilon$ - это частное $s^{-1} \circ j$, в котором $j$ - это вложение подкомплекса $K$ в комплекс $C(p)$ с компонентами $K^{n} \oplus M^{n+1}$ и с дифференциалом

$$
d_{C(p)}=\left(\begin{array}{cc}
d_{K} & p \\
0 & -d_{M}
\end{array}\right)
$$

а квазиизоморфизм $s: N[1] \longrightarrow C(p)$ - это морфизм $(0, i)$.

Выделенный треугольник в $\mathbf{D}^{b}(\mathscr{A})$ - это последовательность в $\mathbf{D}^{b}(\mathscr{A})$ вида

$$
X \stackrel{u}{\longrightarrow} Y \stackrel{v}{\longrightarrow} Z \stackrel{w}{\longrightarrow} X[1]
$$

которая изоморфна стандартному треугольнику.

Пусть $\mathscr{A}$ и $\mathscr{B}$ - две абелевы категории и $F: \mathscr{A} \longrightarrow \mathscr{B}$ - аддитивный функтор, точньй слева (соответственно справа). Функтор $F$ индуцирует функтор между категориями комплексов и функтор $\bar{F}: \mathbf{H}^{b}(\mathscr{A}) \rightarrow \mathbf{H}^{b}(\mathscr{B})$, которые получаются покомпонентным применением $F$. Если $F$ не является точньм, то он не переводит квазиизоморфизмы в квазиизоморфизмы. Тем не менее, в большинстве случаев, можно определить правый (соответственно левый) производньй функтор $\mathbf{R} F$ (соответственно $\mathbf{L} F$ ) между соответствуюшими производными категориями. Производный функтор $\mathbf{R} F$ (соответственно $\mathbf{L} F$ ) будет точным функтором между триангулированньми категориями в следующем смысле: он коммутирует с функторами сдвига и переводит выделенные треугольники в выделенные. Мы не будем приводить здесь определение производных функторов, идея состоит в покомпонентном применении функтора $F$ к правильно 
выбранным представителям классов квазиизоморфных комплексов (см. [43], [15], [12], $[21],[23])$.

В качестве примеров рассмотрим производные категории когерентных пучков на гладких проективных (или собственных алгебраических) многообразиях. Обозначим $\mathbf{D}^{b}(\operatorname{coh}(X))$ через $\mathbf{D}^{b}(X)$. Каждьй морфизм $f: X \rightarrow Y$ индуцирует функтор обратного образа $f^{*}: \operatorname{coh}(Y) \longrightarrow \operatorname{coh}(X)$. Этот функтор является точным справа и имеет левый производный функтор $\mathbf{L} f^{*}: \mathbf{D}^{b}(Y) \longrightarrow \mathbf{D}^{b}(X)$. Для его определения нужно заменить комплекс на $Y$ квазиизоморфным комплексом из локально свободных пучков и применить покомпонентно функтор $f^{*}$ к этому комплексу. Подобньмм же образом для всякого комплекса $\mathscr{F} \in \mathbf{D}^{b}(X)$ можно определить точный функтор $\stackrel{\mathbf{L}}{\otimes}: \mathbf{D}^{b}(X) \longrightarrow \mathbf{D}^{b}(X)$, заменяя $\mathscr{F}$ квазиизоморфным комплексом из локально свободных пучков.

Морфизм $f: X \longrightarrow Y$ индуцирует также функтор прямого образа $f_{*}: \operatorname{coh}(X) \longrightarrow$ $\operatorname{coh}(Y)$, который точен слева и имеет правьй производньй функтор $\mathbf{R} f_{*}: \mathbf{D}^{b}(X) \longrightarrow$ $\mathbf{D}^{b}(Y)$. Для его определения категория когерентных пучков вкладьвается в категорию квазикогерентных пучков, а комплекс заменяется на квазиизоморфньй ему комплекс из инъективных пучков. После этого функтор $f_{*}$ можно применять покомпонентно к комплексу из инъективных пучков. Функтор $\mathbf{R} f_{*}$ сопряжен справа к $\mathbf{L} f^{*}$. Это означает, что существуют функториальные изоморфизмы

$$
\operatorname{Hom}\left(A, \mathbf{R} f_{*} B\right) \cong \operatorname{Hom}\left(\mathbf{L} f^{*} A, B\right)
$$

для всех $A, B$. Данное свойство может также рассматриваться в качестве определения функтора $\mathbf{R} f_{*}$.

Используя данные функторы, можно ввести больший класс точных функторов. Пусть $X$ и $Y$-гладкие проективные (или собственные алгебраические) многообразия. Рассмотрим проекции

$$
X \stackrel{p}{\longleftarrow} X \times Y \stackrel{q}{\longrightarrow} Y .
$$

Каждый объект $\mathscr{E} \in \mathbf{D}^{b}(X \times Y)$ задает точный функтор $\Phi_{\mathscr{E}}: \mathbf{D}^{b}(X) \longrightarrow \mathbf{D}^{b}(Y)$ по следуюшей формуле:

$$
\Phi_{\mathscr{E}}(\cdot):=\mathbf{R} q_{*}\left(\stackrel{\mathscr{E}}{\mathscr{\mathbf { L }}} p^{*}(\cdot)\right) .
$$

Очевидно, что тот же самый объект задает другой функтор $\Psi_{\mathscr{E}}: \mathbf{D}^{b}(Y) \longrightarrow \mathbf{D}^{b}(X)$ по такой же формуле

$$
\Psi \mathscr{E}(\cdot):=\mathbf{R} p_{*}\left(\mathscr{E} \otimes q^{\mathbf{L}} q^{*}(\cdot)\right) .
$$

Таким образом, получается достаточно большой класс точных функторов между ограниченными производными категориями когерентных пучков на гладких проективных многообразиях, класс, состоящий из функторов, имеюших вид $\Phi_{\mathscr{E}}$ для некоторого комплекса $\mathscr{E}$ на произведении многообразий. Данный класс замкнут относительно композиции функторов. Действительно, пусть $X, Y, Z$ - три гладких проективных многообразия, и пусть

$$
\Phi_{I}: \mathbf{D}^{b}(X) \longrightarrow \mathbf{D}^{b}(Y), \quad \Phi_{J}: \mathbf{D}^{b}(Y) \longrightarrow \mathbf{D}^{b}(Z)
$$


- два функтора, где $I$ и $J$-объекты $\mathbf{D}^{b}(X \times Y)$ и $\mathbf{D}^{b}(Y \times Z)$ соответственно. Обозначим через $p_{X Y}, p_{Y Z}, p_{X Z}$ проекции $X \times Y \times Z$ на соответствуюшие пары сомножителей. Композиция $\Phi_{J} \circ \Phi_{I}$ изоморфна $\Phi_{K}$, где $K \in \mathbf{D}^{b}(X \times Z)$ определяется формулой

$$
K \cong \mathbf{R} p_{X Z *}\left(p_{Y Z}^{*}(J) \otimes p_{X Y}^{*}(I)\right) .
$$

Предположительно, класс точных функторов, описанньй вьше, содержит все точные функторы между производньми категориями когерентных пучков на гладких проективных многообразиях. Мы не знаем, верно это или нет, однако это определенно верно для точных эквивалентностей.

Tеорема 3.1 ([33], см. также [35]). Пусть $X$ и $Y$ - гладкие проективные многообразия. Предположим, что $F: \mathbf{D}^{b}(X) \stackrel{\sim}{\longrightarrow} \mathbf{D}^{b}(Y)$ - это точная әквивалентность. Тогда существует единственный ( $с$ точностью до изоморфизма) облект $\mathscr{E} \in \mathbf{D}^{b}(X \times Y)$ такой, что функтор $F$ изоморфен функтору $\Phi_{\mathscr{E}}$.

Теперь рассмотрим ограниченные производные категории когерентных пучков на абелевых многообразиях. Существуют примеры различных абелевых многообразий, которые имеют эквивалентные производные категории когерентных пучков. Более того, можно полностью описать классы абелевых многообразий с эквивалентными производньми категории когерентных пучков.

Пусть $A$ - абелево многообразие размерности $n$ над $\mathbb{C}$. Это означает, что $A-$ комплексный тор $(U / \Gamma, I)$, которьй является алгебраическим, т.е. имеет вложение в проективное пространство. Пусть $\widehat{A}$ - двойственное абелево многообразие, т.е. двойственньй тор $\left(U^{*} / \Gamma^{*},-I^{t}\right)$. Он канонически изоморфен группе Пикара $\operatorname{Pic}^{0}(A)$. Хорошо известно, что существует единственное линейное расслоение $P$ на произведении $A \times \widehat{A}$ такое, что для каждой точки $\alpha \in \widehat{A}$ ограничение $P_{\alpha}$ на $A \times\{\alpha\}$ представляет элемент $\mathrm{Pic}^{0} A$, соответствуюший $\alpha$, и, в дополнение, ограничение $\left.P\right|_{\{0\} \times \widehat{A}}$ тривиально. Такое $P$ назьвается расслоением Пуанкаре.

Расслоение Пуанкаре дает пример точной эквивалентности между производными категориями когерентных пучков двух неизоморфных многообразий. Рассмотрим проекции

$$
A \stackrel{p}{\longleftarrow} A \times \widehat{A} \stackrel{q}{\longrightarrow} \widehat{A}
$$

и функтор $\Phi_{P}: \mathbf{D}^{b}(A) \longrightarrow \mathbf{D}^{b}(\widehat{A})$, определенньй формулой $(9)$, т.е. $\Phi_{P}(\cdot)=$ $\mathbf{R} q_{*}\left(P \otimes p^{*}(\cdot)\right)$. Мукаи доказал [32], что функтор $\Phi_{P}: \mathbf{D}^{b}(A) \longrightarrow \mathbf{D}^{b}(\widehat{A})$ есть точная эквивалентность и сушествует изоморфизм функторов:

$$
\Psi_{P} \circ \Phi_{P} \cong\left(-1_{A}\right)^{*}[n],
$$

где $\left(-1_{A}\right)-$ морфизм взятия обратного в группе $A$.

Пусть $A_{1}$ и $A_{2}$ - два абелевых многообразия одинаковой размерности. Обозначим через $\Gamma_{A_{1}}$ и $\Gamma_{A_{2}}$ решетки первых гомологий $H_{1}\left(A_{1}, \mathbb{Z}\right)$ и $H_{1}\left(A_{2}, \mathbb{Z}\right)$. Каждое отображение $f: A_{1} \longrightarrow A_{2}$ абелевых многообразий индуцирует отображение $\bar{f}: \Gamma_{A_{1}} \longrightarrow \Gamma_{A_{2}}$ на групах первых гомологий.

Для любого абелева многообразия $A$ решетка первых гомологий многообразия $A \times \widehat{A}$ совпадает с $\Gamma_{A} \oplus \Gamma_{A}^{*}$ и, следовательно, обладает канонической симметрической билинейной формой $q_{A}$, определенной по правилу (6). Рассмотрим изоморфизм 
$f: A_{1} \times \widehat{A}_{1} \stackrel{\sim}{\longrightarrow} A_{2} \times \widehat{A}_{2}$ абелевых многообразий. Назовем его изометричныцм, если изоморфизм $\bar{f}: \Gamma_{A_{1}} \oplus \Gamma_{A_{1}}^{*} \stackrel{\sim}{\longrightarrow} \Gamma_{A_{2}} \oplus \Gamma_{A_{2}}^{*}$ отождествляет формы $q_{A_{1}}$ и $q_{A_{2}}$.

Сейчас мы сформулируем критерий того, когда два абелевых многообразия имеют эквивалентные производные категории когерентных пучков.

Теорема 3.2 [34]. Пусть $A_{1}$ и $A_{2}$ - абелевы многообразия. Производные категории $\mathbf{D}^{b}\left(A_{1}\right)$ и $\mathbf{D}^{b}\left(A_{2}\right)$ являются әквивалентными как триангулированные категории тогда и только тогда, когда существует изометричный изоморфизм

$$
f: A_{1} \times \widehat{A}_{1} \stackrel{\sim}{\longrightarrow} A_{2} \times \widehat{A}_{2},
$$

m.е. $\bar{f}$ отождествляет формы $q_{A_{1}}$ и $q_{A_{2}}$ на $\Gamma_{A_{1}} \oplus \Gamma_{\widehat{A}_{1}} u \Gamma_{A_{2}} \oplus \Gamma_{\widehat{A}_{2}}$.

Используя теоремы 2.1 и 3.2 , можно произвести некоторую проверку гипотезы о гомологической зеркальной симметрии для торов. Предположим, что два тора $\left(T_{1}, I_{1}, G_{1}, B_{1}\right)$ и $\left(T_{2}, I_{2}, G_{2}, B_{2}\right)$ оба зеркально симметричны тору $\left(T^{\prime}, I^{\prime}, G^{\prime}, B^{\prime}\right)$. Тогда $\operatorname{SCFT}\left(\Gamma_{1}, I_{1}, G_{1}, B_{1}\right)$ изоморфна $\operatorname{SCFT}\left(\Gamma_{2}, I_{2}, G_{2}, B_{2}\right)$, и по теореме 2.1 существует изоморфизм решеток $\Gamma_{1} \oplus \Gamma_{1}^{*}$ и $\Gamma_{2} \oplus \Gamma_{2}^{*}$, который отождествляет $q_{1}$ и $q_{2}, \mathscr{I}_{1}$ и $\mathscr{I}_{2}$ и $\mathscr{J}_{1}$ и $\mathscr{J}_{2}$.

С другой стороны, если теперь предположить, что оба комплексных тора $\left(T_{1}, I_{1}\right)$ и $\left(T_{2}, I_{2}\right)$ являются алгебраическими, тогда ГЗС влечет эквиваленьность $\mathbf{D}^{b}\left(\left(T_{1}, I_{1}\right)\right)$ и $\mathbf{D}^{b}\left(\left(T_{2}, I_{2}\right)\right)$. Критерий для эквивалентности дается в теореме 3.2 : он требует существования изоморфизма между $\Gamma_{1} \oplus \Gamma_{1}^{*}$ и $\Gamma_{2} \oplus \Gamma_{2}^{*}$, который переводит $q_{1}$ в $q_{2}$ и $\widetilde{\mathscr{I}_{1}}$ в $\widetilde{\mathscr{I}_{2}}$. Очевидно, что так как в общем случае $\mathscr{I} \neq \mathscr{I}$, мы получаем два условия, которые противоречат друг другу. Однако, так как $\mathscr{I}$ совпадает с $\widetilde{\mathscr{I}}$ при условии $B^{(0,2)}=0$, получается следуюший результат.

СлЕДСТвИЕ 3.3. Если $\operatorname{SCFT}\left(\Gamma_{1}, I_{1}, G_{1}, B_{1}\right)$ изоморфна $\operatorname{SCFT}\left(\Gamma_{2}, I_{2}, G_{2}, B_{2}\right)$, оба тора $\left(T_{1}, I_{1}\right)$ и $\left(T_{2}, I_{2}\right)$ алгебраичны и обе формы $B_{1}$ и $B_{2}$ имеют тип $(1,1)$, то $\mathbf{D}^{b}\left(\left(T_{1}, I_{1}\right)\right)$ и $\mathbf{D}^{b}\left(\left(T_{2}, I_{2}\right)\right)$ әквивалентны.

Пусть $\left(T_{1}, I_{1}, G_{1}, B_{1}\right)$ - комплексньй тор с плоской кэлеровой метрикой и В-полем типа $(1,1)$, и пусть $\left(T_{2}, I_{2}\right)$ - другой комплексньй тор. Предположим, что сушествует изоморфизм решеток $g: \Gamma_{1} \oplus \Gamma_{1}^{*} \rightarrow \Gamma_{2} \oplus \Gamma_{2}^{*}$, переводящий $q_{1}$ в $q_{2}$ и $\widetilde{\mathscr{I}}_{1}$ в $\widetilde{\mathscr{I}}_{2}$. В этом случае можно доказать, что на $T_{2}$ существуют кэлерова метрика $G_{2}$ и В-поле $B_{2}$ типа $(1,1)$ такие, что $\operatorname{SCFT}\left(\Gamma_{1}, I_{1}, G_{1}, B_{1}\right)$ и $\operatorname{SCFT}\left(\Gamma_{2}, I_{2}, G_{2}, B_{2}\right)$ изоморфны как $N=2$ суперконформные теории поля.

Комбинируя это с теоремой 2.1 и критерием эквивалентности для производных категорий $\mathbf{D}^{b}\left(\left(T_{1}, I_{1}\right)\right)$ и $\mathbf{D}^{b}\left(\left(T_{2}, I_{2}\right)\right)$, получаем результат, обратный к следствию 3.3.

СлЕДСТВИЕ 3.4. Пусть $\left(T_{1}, I_{1}, G_{1}, B_{1}\right)$ - алгебраический тор с плоской кәлеровой метрикой и $\mathrm{B}$-полем типа $(1,1)$. Пусть $\left(T_{2}, I_{2}\right)$ - другой алгебраический тор. Предположим, что $\mathbf{D}^{b}\left(\left(T_{1}, I_{1}\right)\right)$ әквивалентна $\mathbf{D}^{b}\left(\left(T_{2}, I_{2}\right)\right)$. Тогда на $T_{2}$ найдутся кәлерова метрика $G_{2}$ и $\mathrm{B}$-поле $B_{2}$ типа $(1,1)$ такие, что $\operatorname{SCFT}\left(\Gamma_{1}, I_{1}, G_{1}, B_{1}\right)$ u $\operatorname{SCFT}\left(\Gamma_{2}, I_{2}, G_{2}, B_{2}\right)$ изоморфны как $N=2$ суперконформные теории поля.

Если $\operatorname{dim}_{\mathbb{C}} T=1$, то В-поле автоматически имеет тип $(1,1)$. Следовательно, в этом случае ГЗС выдерживает проверку. Конечно, это то, что мы ожидаем, так как известно, что ГЗС для эллиптических кривых верна [39]. С другой стороны, кажется, что в случае $\operatorname{dim}_{\mathbb{C}} T>1$ имеются проблемы. 
Однако не все потеряно - и в этом случае существует простая модификация ГЗС, которая проходит наше тестирование. Эта модификация подразумевает замену $\mathbf{D}^{b}((T, I))$ производной категорией $\beta(B)$-скрученных пучков, где $\beta(B)$ - это элемент $H^{2}\left((T, I), \mathscr{O}_{T}^{*}\right)$, зависящий от В-поля $B \in H^{2}(X, \mathbb{R})$.

Пусть $X$ - алгебраическое многообразие над $\mathbb{C}$, и пусть $B \in H^{2}(X, \mathbb{R})$. Рассмотрим гомоморфизм $\beta: H^{2}(X, \mathbb{R}) \rightarrow H^{2}\left(X, \mathscr{O}_{X}^{*}\right)$, индуцированный отображением $\mathbb{R} \longrightarrow \mathscr{O}_{X}^{*}$, которое получается из следуюшей коммутативной диаграммы:

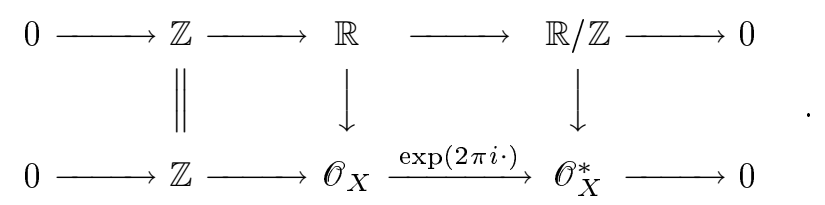

Каждьй элемент $a \in H^{2}\left(X, \mathscr{O}_{X}^{*}\right)$ задает $\mathscr{O}_{X}^{*}$-джерб $\mathscr{X}_{a}$ на $X$. Рассмотрим категорию $\operatorname{coh}_{1}\left(\mathscr{X}_{a}\right)$ когерентных пучков веса 1 на джербе $\mathscr{X}_{a}$. Теперь наша триангулированная категория может быть определена как производная категория $\mathbf{D}^{b}\left(\operatorname{coh}_{1}\left(\mathscr{X}_{\beta(B)}\right)\right)$ и будет обозначаться $\mathbf{D}^{b}(X, B)$. Напомним, что когерентные пучки веса 1 на джербе $\mathscr{X}_{a}$ могут быть определены как скрученные когерентные пучки на $X$ в следующем смысле. Выберем открытое покрытие $\left\{U_{i}\right\}_{i \in I}$ многообразия $X$, для которого элемент $a \in H^{2}\left(X, \mathscr{O}_{X}^{*}\right)$ представляется 2-коциклом Чеха $a_{i j k} \in \Gamma\left(U_{i j k}, \mathscr{O}_{X}^{*}\right)$, где $U_{i j k}=U_{i} \cap U_{j} \cap U_{k}$. Теперь $a$-скрученный пучок можно определить как набор когерентных пучков $\mathscr{F}_{i}$ на $U_{i}$ для всех $i \in I$ вместе с изоморфизмами $\phi_{j i}:\left.\left.\mathscr{F}_{i}\right|_{U_{i j}} \stackrel{\sim}{\longrightarrow} \mathscr{F}_{j}\right|_{U_{i j}}$ для всех пар $i, j \in I$ ( $\left.\phi_{i j}=\phi_{j i}^{-1}\right)$, удовлетворяющими скрученному условию коцикла $\phi_{i j} \phi_{j k} \phi_{k i}=a_{i j k} \mathrm{id}$.

В случае, когда $\beta(B)$ - это элемент кручения в $H^{2}\left(X, \mathscr{O}_{X}^{*}\right)$, абелева категория скрученных пучков эквивалентна абелевой категории когерентных пучков модулей над пучком алгебр Адзумая $\mathscr{A}_{B}$, который соответствует данному элементу. Это влечет также эквивалентность соответствующих производных категорий.

Напомним определение и основные факты об алгебрах Адзумая. Пусть $\mathscr{A}$ - это $\mathscr{O}_{X}$-алгебра, которая когерентна как пучок $\mathscr{O}_{X}$-модулей. Она назьвается алгеброй Адзумая, если она является локально свободньм пучком $\mathscr{O}_{X}$-модулей и для каждой точки $x \in X$ ограничение $\mathscr{A}(x):=\mathscr{A} \otimes_{\mathscr{O}_{X}} \mathbb{C}(x)$ изоморфно матричной алгебре $M_{r}(\mathbb{C})$. Тривиальная алгебра Адзумая - это алгебра вида $\mathscr{E} n d(E)$, где $E$-векторное расслоение. Две алгебры Адзумая $\mathscr{A}$ и $\mathscr{A}^{\prime}$ назьваются подобными (или эквивалентными по Морите), если найдутся векторные расслоения $E$ и $E^{\prime}$ такие, что

$$
\mathscr{A} \otimes_{\mathscr{O}_{X}} \mathscr{E} n d(E) \cong \mathscr{A}^{\prime} \otimes_{\mathscr{O}_{X}} \mathscr{E} n d\left(E^{\prime}\right) .
$$

Обозначим через $\operatorname{coh}(\mathscr{A})$ абелеву категорию пучков (правых) $\mathscr{A}$-модулей, которые когерентны как пучки $\mathscr{O}_{X}$-модулей, а через $\mathbf{D}^{b}(X, \mathscr{A})$ ограниченную производную категорию $\operatorname{coh}(\mathscr{A})$. Легко видеть, что если алгебры Адзумая $\mathscr{A}$ и $\mathscr{A}^{\prime}$ подобны, то категории $\operatorname{coh}(\mathscr{A})$ и $\operatorname{coh}\left(\mathscr{A}^{\prime}\right)$ эквивалентны и, значит, производные категории $\mathbf{D}^{b}(\mathscr{A})$ и $\mathbf{D}^{b}\left(\mathscr{A}^{\prime}\right)$ также эквивалентны.

Алгебры Адзумая по модулю эквивалентности по Морите образуют группу относительно тензорного умножения. Эта группа назьвается группй Брауэра многообразия $X$ и обозначается $\operatorname{Br}(X)$. Сушествует естественное отображение

$$
\operatorname{Br}(X) \longrightarrow H^{2}\left(X, \mathscr{O}_{X}^{*}\right) .
$$


Это отображение является вложением, а его образ содержится в подгруппе кручения $H^{2}\left(X, \mathscr{O}_{X}^{*}\right)_{t o r s}$. Последняя группа обозначается $B r^{\prime}(X)$ и назьвается когомологической группой Брауэра $X$. Хорошо известная гипотеза Гротендика утверждает, что естественное отображение $B r(X) \longrightarrow B r^{\prime}(X)$ есть изоморфизм для гладких многообразий. Эта гипотеза была доказана для абелевых многообразий [16].

Предположим, что $\beta(B)$ - элемент кручения в $H^{2}\left((T, I), \mathscr{O}_{T}^{*}\right)$, и рассмотрим алгебру Адзумая $\mathscr{A}_{B}$, соответствуюшую этому элементу. Производная категория $\mathbf{D}^{b}\left((T, I), \mathscr{A}_{B}\right)$ не зависит от выбора $\mathscr{A}_{B}$, так как все такие алгебры эквивалентны по Морите. Можно показать, что производная категория $\mathbf{D}^{b}((T, I), B)$ эквивалентна категории $\mathbf{D}^{b}\left((T, I), \mathscr{A}_{B}\right)$.

Достаточное условие в случае алгебраических торов для эквивалентности категорий $\mathbf{D}^{b}\left(\left(T_{1}, I_{1}\right), B_{1}\right)$ и $\mathbf{D}^{b}\left(\left(T_{2}, I_{2}\right), B_{2}\right)$ дается следующей теоремой [38].

TeOpema 3.5 [38]. Пусть $\left(T_{1}, I_{1}\right)$ и $\left(T_{2}, I_{2}\right)$ - два алгебрачческих тора. Пусть $B_{1} \in H^{2}\left(T_{1}, \mathbb{R}\right)$ и $B_{2} \in H^{2}\left(T_{2}, \mathbb{R}\right)$ отображаются посредством $\beta$ в әлементы кручения. Если найдется изоморфизм решеток $\Gamma_{1} \oplus \Gamma_{1}^{*} u \Gamma_{2} \oplus \Gamma_{2}^{*}$, переводящий $q_{1}$ в $q_{2}$, а $\mathscr{I}_{1}$ в $\mathscr{I}_{2}$, то категории $\mathbf{D}^{b}\left(\left(T_{1}, I_{1}\right), B_{1}\right)$ и $\mathbf{D}^{b}\left(\left(T_{2}, I_{2}\right), B_{2}\right)$ будут әквивалентны.

Представляется вероятньм, что данное условие также является и необходимым для того, чтобы $\mathbf{D}^{b}\left(\left(T_{1}, I_{1}\right), B_{1}\right)$ была эквивалентна $\mathbf{D}^{b}\left(\left(T_{2}, I_{2}\right), B_{2}\right)$. Комбинируя теорему 3.5 с нашей теоремой 2.1 , получаем такой результат.

СлЕДСтвИЕ 3.6. Пусть $\operatorname{SCFT}\left(\Gamma_{1}, I_{1}, G_{1}, B_{1}\right)$ u $\operatorname{SCFT}\left(\Gamma_{2}, I_{2}, G_{2}, B_{2}\right)$ изоморфнье, оба тора $\left(T_{1}, I_{1}\right)$ и $\left(T_{2}, I_{2}\right)$ алгебраичны и как $B_{1}$, так и $B_{2}$ отображаются посредством $\beta$ в әлементы кручения. Тогда категории $\mathbf{D}^{b}\left(\left(T_{1}, I_{1}\right), B_{1}\right)$ и $\mathbf{D}^{b}\left(\left(T_{2}, I_{2}\right), B_{2}\right)$ әквивалентны.

Данное следствие подсказьвает способ модифицировать ГЗС - заменить $\mathbf{D}^{b}(X)$ на $\mathbf{D}^{b}(X, B)$. Напомним, что наличие В-поля изменяет и определение категории $\Phi$ кая, которая обозначается в этом случае через $\mathbf{D} \mathscr{F}_{0}(X, B)$. Модифицированная ГЗС утверждает, что если $(X, G, B)$ и $\left(X^{\prime}, G^{\prime}, B^{\prime}\right)$ зеркально симметричны, то $\mathbf{D}^{b}(X, B)$ эквивалентна $\mathbf{D} \mathscr{F}_{0}\left(X^{\prime}, B^{\prime}\right)$. Следствие 3.6 показывает, что эта гипотеза выдерживает проверку, в то время как оригинальная ГЗС нарушается.

Для эллиптических кривых модифицированная ГЗС не сильно отличается от оригинальной. Так как в этом случае $h^{0,2}=0$, то комплексная сторона никак не изменяется с введением В-поля, в то время как на симплектической стороне единственным эффектом является комплексификация симплектической формы (при замене $\omega$ на $\omega+i B$ ). Для настояших многообразий Калаби-Яу $h^{0,2}$ также зануляется, и комплексная сторона снова не изменяется, но на симплектической стороне наличие В-поля приводит к радикальным изменениям. К примеру, плоские связности на лагранжевых подмногообразиях должны быть заменены на проективно плоские, что ведет к уменьшению количества $A$-бран. А вот для комплексных торов размерности больше единицы В-поле сильно влияет как на $A$-браны, так и на $B$-браны.

\section{4. Категория $A$-бран и категория Фукая}

В этой лекции мы обсудим $D$-браны типа $A$ ( $A$-браны) на многообразиях Калаби-Яу. Как уже говорилось вьше, множество $A$-бран имеет структуру аддитивной 
категории, и если $X$ зеркально $X^{\prime}$, то категория $A$-бран на $X$ эквивалентна категории $B$-бран на $X^{\prime}$, и наоборот. Можно считать установленным, что категория $B$-бран на многообразиях Калаби-Яу $X$ эквивалентна категории $\mathbf{D}^{b}(X)^{5}[7],[28],[1],[5],[22]$. Гипотеза ГЗС поэтому сводится к утверждению, что категория $A$-бран на $X$ эквивалентна производной категории Фукая на $X$. Как мы увидим, последнее утверждение неверно для некоторых $X$, так что гипотеза ГЗС нуждается в модификации.

В случае, когда $X$ - эллиптическая кривая, гипотеза ГЗС (с небольшими модификациями) была доказана в работе [39]. С другой стороны, в работе [20] было показано, что, вообще говоря, производная категория Фукая является только полной подкатегорией категории $A$-бран. В случае, когда $X$ - тор размерности больше 2 с постоянной симплектической структурой, в работе [20] были построены примеры $A$-бран, которые являются коизотропными, а не лагранжевыми подмногообразиями в $X$. Пока неясно, как дать математически строгое определение категории $A$-бран. В этой лекции мы опишем результаты работы [20] и обсудим нерешенные проблемы.

Чтобы продемонстрировать, что категория $A$-бран на многообразии $X$, вообше говоря, "больше" $\mathbf{D} \mathscr{F}_{0}(X)$, мы предъявим зеркальную пару $X$ и $X^{\prime}$ такую, что группа $K^{0}\left(\mathbf{D}^{b}(X)\right)$ строго больше, чем $K^{0}\left(\mathbf{D} \mathscr{F}_{0}\left(X^{\prime}\right)\right)$. Для простоты, можно еше тензорно умножить $K^{0}$ на $\mathbb{Q}$ и применить характер Черна, который принимает значения в когомологиях $X$. В случае $\mathbf{D}^{b}(X)$ характер Черна сh принимает значения в пересечении $H^{*}(X, \mathbb{Q})$ и $\bigoplus_{p=0}^{n} H^{p, p}(X)$, которые рассматриваются как подгруппы $H^{*}(X, \mathbb{C})$. (Согласно гипотезе Ходжа, образ ch совпадает с этим пересечением.) В случае категории Фукая зеркального многообразия $X^{\prime}$ можно сказать следующее. Во-первых, зеркальная симметрия индуцирует изоморфизм $H^{*}(X, \mathbb{C})$ и $H^{*}\left(X^{\prime}, \mathbb{C}\right)$, так что аналог характера Черна для категории Фукая должен принимать значения в какой-нибудь подгрупе $H^{*}\left(X^{\prime}, \mathbb{C}\right)$. Естественно предположить, что характер Черна объекта категории Фукая $(Y, E, \nabla)$ - это класс когомологий (над $\mathbb{Q})$, дуальный по Пуанкаре гомологическому классу $Y$. Эту гипотезу можо обосновать физическими соображениями, и мы будем считать, что она верна.

Пусть $E$ - эллиптическая кривая, $e$ - произвольная точка на $E$, a $\operatorname{End}_{e}(E)$ - кольцо эндоморфизмов $E$, сохраняюших $e$. Для большинства $E$ это кольцо изоморфно $\mathbb{Z}$, но для некоторых специальных $E$ кольцо $\operatorname{End}_{e}(E)$ строго больше, чем $\mathbb{Z}$. Такие кривые называются эллиптическими кривыми с комплексным умножением. Нетрудно проверить, что $E$ обладает комплексньм умножением тогда и только тогда, когда ее параметр Тейхмюллера $\tau$ - корень квадратного уравнения с целыми коэффициентами. Пусть $E$ - эллиптическая кривая с комплексным умножением. Рассмотрим абелево многообразие $X=E^{n}, n \geqslant 2$. Можно показать, что для такого многообразия образ характера Черна

$$
\operatorname{ch}: K^{0}\left(\mathbf{D}^{b}(X)\right) \otimes \mathbb{Q} \longrightarrow H^{*}(X, \mathbb{Q})
$$

совпадает с пересечением

$$
H^{*}(X, \mathbb{Q}) \cap\left(\bigoplus_{p=0}^{n} H^{p, p}(X)\right)
$$

и имеет размерность

$$
\operatorname{dim}_{\mathbb{Q}} \operatorname{Im}(\mathrm{ch})=\left(\begin{array}{c}
2 n \\
n
\end{array}\right) .
$$

\footnotetext{
${ }^{5}$ В этой лекции для простоты предполагается, что В-поле равно нулю.
} 
Далее, абелево многообразие $X$ зеркально некоторому симплектическому тору $X^{\prime}$ вещественной размерности $2 n$. Любой класс когомологий $X^{\prime}$, дуальный по Пуанкаре лагранжеву подмногообразию в $X^{\prime}$, принадлежит ядру отображения

$$
H^{n}\left(X^{\prime}, \mathbb{R}\right) \stackrel{\wedge \omega}{\longrightarrow} H^{n+2}\left(X^{\prime}, \mathbb{R}\right) .
$$

Это отображение - эпиморфизм, поэтому его размерность равна $\left(\begin{array}{c}2 n \\ n\end{array}\right)-\left(\begin{array}{c}2 n \\ n+2\end{array}\right)$. Следовательно, образ характера Черна категории Фукая не превосходит $\left(\begin{array}{c}2 n \\ n\end{array}\right)-\left(\begin{array}{c}2 n \\ n+2\end{array}\right)$. $\mathrm{C}$ другой стороны, зеркальная симметрия дает нам изоморфизм между когомологиями $X$ и $X^{\prime}$ [13]. Если сделать резонное допущение, что этот изоморфизм согласован с эквивалентностью категорий $B$-бран на $X$ и $A$-бран на $X^{\prime}$, то можно заключить, что категория $\mathbf{D} \mathscr{F}_{0}\left(X^{\prime}\right)$ не может быть эквивалентна категории $A$-бран на $X^{\prime} . \mathrm{C}$ другой стороны, мы знаем из физических соображений, что первая категория является полной подкатегорией второй.

Возникает вопрос: если не все $A$-браны происходят из лагранжевых подмногообразий, то как правильно описать их геометрию? На когомологическом уровне этот вопрос сводится к следующему: если характер Черна не обязательно лежит в ядре отображения (10), то где он принимает значения? Ответ на последний вопрос можно описать так [13], [18]. Пусть на $X$ задана какая-нибудь постоянная кэлерова форма такая, что $X=A \times B$, где $A$ и $B$ - лагранжевы вешественные подторы. Тогда тор $X^{\prime}=\widehat{A} \times B$ зеркален $X$. Рассмотрим тор $Z=A \times \widehat{A} \times B$. У него есть две очевидные проекции $\pi$ и $\pi^{\prime}$ на $X$ и $X^{\prime}$. На $A \times \widehat{A}$ у нас также есть расслоение Пуанкаре $P$, характер Черна которого мы обозначим $\operatorname{ch}(P)$. Используя очевидную проекцию $Z$ на $A \times \widehat{A}$, можно рассматривать $\operatorname{ch}(P)$ как класс когомологий на $Z$. Пусть у нас теперь есть какой-нибудь класс когомологий $\alpha \in H^{*}(X, \mathbb{Q})$. Опишем его образ при зеркальной симметрии [13]. Надо взять обратньй образ $\alpha$ по отношению к отображению $\pi: Z \rightarrow X$, тензорно умножить на $\operatorname{ch}(P)$, а потом взять прямой образ при отображении $\pi^{\prime}: Z \rightarrow X^{\prime}$. Получается класс $\alpha^{\prime} \in H^{*}\left(X^{\prime}, \mathbb{Q}\right)$, которьй является образом $\alpha$ при зеркальной симметрии. Можно показать, что если $\alpha$ принадлежит пересечению $H^{*}(X, \mathbb{Q})$ и $\bigoplus_{p} H^{p, p}(X)$, то $\alpha^{\prime}$ удовлетворяет

$$
\iota_{\omega^{-1}} \alpha^{\prime}-\omega \wedge \alpha^{\prime}=0
$$

3 десь $\iota_{\omega^{-1}}$ - оператор внутреннего умножения на бивектор $\omega^{-1}$. Классы когомологий, дуальные лагранжевым подмногообразиям, удовлетворяют этому уравнению, но оно имеет и другие решения. Например, легко построить примеры решений вида $\alpha^{\prime}=e^{a}$, где $a \in H^{2}\left(X^{\prime}, \mathbb{Z}\right)$. Такие примеры наводят на мысль, что для некоторых симплектических многообразий $X$ существуют линейные расслоения на $X$, которые являются $A$-бранами. Мы вскоре увидим, что это действительно так.

Чтобы лучше понять геометрию $A$-бран, обратимся к физике. Как уже объяснялось в лекции 1 , классическая $A$-брана - это граничное условие для сигма-модели, которое сохраняет $N=2$ супералгебру Вирасоро. В работе [20] мы проанализировали это условие в предположении, что $A$-брана описьвается следуюшими геометрическими данными: подмногообразием $Y$ в $X$, линейньм расслоением $E$ на $Y$, снабженном эрмитовой метрикой, и унитарной связностью $\nabla_{E}$ на $E$. Мы показали, что 
тройка $\left(Y, E, \nabla_{E}\right)$ задает $A$-брану тогда и только тогда, когда выполнены следуюшие три условия.

(a) $Y$ - коизотропное подмногообразие в $X$. Это означает, что ограничение симплектической формы $\omega$ на $Y$ - форма постоянного ранга, а ее ядро - интегрируемое распределение $\mathscr{L} Y \subset T Y$. (Эквивалентное определение: $Y$ коизотропно, если для любой точки $p \in Y$ косоортогональное дополнение к подпространству $T Y_{p} \subset T X_{p}$ содержится в $T Y_{p}$.) Фактор-расслоение $T Y / \mathscr{L} Y$ будет обозначаться $\mathscr{N} Y$. Из определения коизотропности следует, что форма $\left.\omega\right|_{Y}$ индуцирует на векторном расслоении $\mathscr{N} Y$ симплектическую форму, которую мы будем обозначать $\sigma$.

(б) 2-форма кривизны $F=(2 \pi i)^{-1} \nabla_{E}^{2}$, рассматриваемая как отображение из векторного расслоения $T Y$ в векторное расслоение $T Y^{*}$, зануляется на подрасслоении $\mathscr{L} Y$. (Фактор $(2 \pi i)^{-1}$ в определении $F$ введен для того, чтобы $F$ была вещественной 2-формой с целочисленными периодами.) Это означает, что 2-форма $F$ индуцирует на $\mathcal{N} Y$ кососимметричное спаривание, которое мы будем обозначать $f$.

(в) Формы $\sigma$ и $f$, рассматриваемые как отображения из $\mathscr{N} Y$ в $\mathscr{N} Y^{*}$, удовлетворяют уравнению $\left(\sigma^{-1} f\right)^{2}=-\mathrm{id}_{\mathcal{N}_{Y}}$. Это значит, что тензор $\mathscr{J}=\sigma^{-1} f$ является комплексной структурой на расслоении $\mathcal{N} Y$.

Прокомментируем эти условия. Из условия (а) вытекает, что на $Y$ имеется структура слоения, размерность которого равна коразмерности $Y$ в $X$. Это слоение назьвается характеристическим слоением коизотропного подмногообразия $Y$. Если характеристическое слоение $Y$ является локально тривиальным расслоением $p: Y \rightarrow Z$ с гладкой базой $Z$, то $\mathscr{N} Y$ - это просто обратный образ касательного расслоения $T Z$ : $\mathscr{N} Y=p^{*} T Z$, а кососимметрическая форма $\sigma$ - обратньй образ некоторой симплектической формы на $Z$. В обшем случае мы имеем дело с многообразием $Y$ со слоением и векторным расслоением $\mathscr{N} Y$ на нем, а пространство слоев $Z$ этого слоения не является гладким многообразием, или даже хаусдорфовым топологическим пространством. Тем не менее, имеет смысл рассматривать пучок гладких сечений расслоения $\mathscr{N} Y$, локально постоянных вдоль слоев слоения. Этот пучок играет роль обратного образа пучка гладких сечений несуществующего, вообще говоря, касательного расслоения к $Z$. Удобно также думать про форму $\sigma$ как симплектическую форму на $Z$. В теории слоений эту ситуацию выражают словами " $\sigma$ - трансверсальная симплектическая форма на многообразии $Y$ со слоением”. Векторное расслоение $\mathscr{N} Y$ обычно называют нормальным расслоением многообразия $Y$ со слоением (его не следует путать с нормальным расслоением к самому $Y$ ).

Условие (б) означает, что для любого сечения $v$ расслоения $\mathscr{L} Y$ мы имеем $\iota_{v} F=0$. Поскольку $d F=0$, то производная Ли от $F$ вдоль $v$ равна нулю, т. е. форма $F$ постоянна вдоль слоев слоения. Если характеристическое слоение - это локально тривиальное расслоение с гладкой базой $Z$, то это означает, что 2 -форма $f$ - обратный образ замкнутой 2-формы на $Z$. В общем случае можно сказать, что $f$ - трансверсально-замкнутая форма на многообразии $Y$ со слоением.

Условие (в), прежде всего, говорит нам, что 2-форма $f$ невырождена. Таким образом, $f$-трансверсальная симплектическая форма на $Y$, в точности как и $\sigma$. Во-вторых, это условие говорит нам, что $\mathscr{J}=\sigma^{-1} f$ - это трансверсальная квазикомплексная структура на многообразии $Y$ со слоением. Если слоение является локально триви- 
альньм расслоением с гладкой базой $Z$, то $\mathscr{J}$ - это просто квазикомплексная структура на $Z$.

Из этих трех условий легко вытекает, что размерность $Y$ должна иметь вид $n+2 k$, где $n=\frac{1}{2} \operatorname{dim}_{\mathbb{R}} X$, а $k$ - неотрицательное целое число. Число $k$ имеет смысл “трансверсальной комплексной размерности $Y$ ”. Если $k=0$, то условие (а) говорит, что многообразие $Y$ лагранжево, и тогда условие (б) требует $F=0$. (Условие (в) в этом случае пусто.) Другой интересньй частный случай - это когда $Y=X$ (это возможно только если $n$ четно). В этом случае слои слоения - точки, условия (а) и (б) вьполняются автоматически, а условие (в) означает, что эндоморфизм $\omega^{-1} F-$ квазикомплексная структура на $X$.

Менее очевидно, что трансверсальная квазикомплексная структура $\mathscr{J}$ интегрируема [20]. Это легко следует из теоремы Гельфанда-Дорфман, которая играет важную роль в теории интегрируемых систем [11]. Таким образом, $Y$ - трансверсально-комплексное многообразие. Легко видеть, что формы $f$ и $\sigma$ имеют тип $(0,2)+(2,0)$ по отношению к $\mathscr{J}$. Более того, форма $f+i \sigma-$ трансверсальная голоморфная симплектическая форма на трансверсально-комплексном многообразии $Y$.

Несколько загадочное условие (в) можно выразить различными способами. Например, оно эквивалентно следующему набору условий:

$$
\wedge^{r}(f+i \sigma) \neq 0, \quad r<k, \quad \wedge^{k}(f+i \sigma)=0 .
$$

Тут $k$ такое же, как и вьше.

Попытки обобщить условия (а)-(в) на случай, когда векторное расслоение $E$ имеет ранг вьше единицы, не увенчались полным успехом. Условия (а) и (б) легко обобщаются, но с условием (в) - проблемы. Физические и математические соображения указьвают, что правильное обобщение условия (в) вьплядит так:

$$
\left(\sigma^{-1} f\right)^{2}=-\operatorname{id}_{E \otimes \mathscr{N} Y}
$$

Тут “трансверсальная форма кривизны” $f$ рассматривается как сечение расслоения $\operatorname{End}(E) \otimes \Lambda^{2}\left(\mathcal{N} Y^{*}\right)$. Из такого условия никакой трансверсальной комплексной структуры на $Y$ не получается, и его геометрический смысл непонятен. Это ведет к трудностям с определением морфизмов между такими $A$-бранами (см. ниже).

До сих пор мы обсуждали $A$-браны на классическом уровне. На квантовом уровне $N=2$ супералгебра Вирасоро может быть аномальной. Условие отсутствия аномалии - важное дополнительное условие на $A$-браны. Рассмотрим, например, $R$-симметрию $J$, компоненты Фурье которой обозначались ранее $J_{n}$ и $\bar{J}_{n}$. Эта симметрия, вообше говоря, имеет аномалию из-за струнных инстантонов, т.е. из-за вкладов в интеграл по траекториям струны, происходящих из дисков в $X$ с границей на $Y$, которые не могут быть продеформированы в точку. В случае, когда $Y$ - лагранжево подмногообразие $X$, аномалии $R$-симметрии были проанализированы К. Хори [17]. Оказывается, что аномалии отсутствуют тогда и только тогда, когда зануляется класс Маслова подмногообразия $Y$. Это объясняет, почему в определении категории Фукая требуется, чтобы объекты были лагранжевыми подмногообразиями с нулевым классом Маслова.

Для более обших коизотропных $A$-бран условие отсутствия аномалий было получено в работе [30]. Пусть $F-2$-форма кривизны линейного расслоения $E$ на $Y$, и пусть 
размерность $Y$ будет равна $n+2 k$, как и выше. Пусть $\Omega$ - голоморфная тривиализация канонического линейного расслоения на $X$. Можно показать, что $(n+2 k)$-форма $\left.\Omega\right|_{Y} \wedge F^{k}$ нигде не зануляется, а потому имеет место равенство $\left.\Omega\right|_{Y} \wedge F^{k}=h \cdot \mathrm{vol}$, где vol - форма объема на $Y$, а $h$ - гладкая комплекснозначная функция на $Y$, которая нигде не обрашается в нуль. Можно рассматривать $h$ как элемент $H^{0}\left(Y, \mathscr{C}_{Y}^{\infty *}\right)$, где $\mathscr{C}_{Y}^{\infty}$ - пучок гладких $\mathbb{C}^{*}$-значных функций на $Y$. Пусть $\alpha_{h}$ - элемент $H^{1}(Y, \mathbb{Z})$, которьй является образом $h$ при гомоморфизме Бокштейна. Аномалии отсутствуют тогда и только тогда, когда $\alpha_{h}=0$.

Если $X$ - тор с постоянной симплектической формой, $Y$ - его аффинньй подтор и 2-форма $F$ постоянна, то квантование сигма-модели с границами проводится до конца, и легко проверить, что супералгебра Вирасоро “выживает" и на квантовом уровне. Это доказьвает, что коизотропные $A$-браны существуют на квантовом уровне.

Вьшеизложенные аргументы должны убедить читателя, что $A$-браны могут не быть лагранжевыми подмногообразиями и что категория Фукая должна быть пополнена более общими коизотропными $A$-бранами. $\mathrm{K}$ сожалению, мы не можем пока сформулировать математически строгое определение категории $A$-бран, которое обобщало бы фукаевское определение. В оставшейся части этой лекции мы обсудим одну эвристическую идею в этом направлении.

В этих лекциях мы встречались с двумя типами $A$-бран. Во-первых, мы обсуждали объекты категории Фукая, т.е. тройки $\left(Y, E, \nabla_{E}\right)$, где $Y$ - лагранжево подмнообразие, $E$ - эрмитово векторное расслоение на $Y$, а $\nabla_{E}-$ плоская унитарная связность на $E$. Во-вторых, мы имели дело с тройками вида $\left(Y, E, \nabla_{E}\right)$, где $E$ - эрмитово линейное расслоение на $Y, \nabla_{E}$ - унитарная связность на $E$ и вьполнены условия (а)-(в). Для объектов категории Фукая мы знаем, в принципе, как вычислять пространства морфизмов и их композиции. Попробуем угадать, как это сделать для объектов второго типа.

Мы должны придумать обобщение комплекса Флоера на коизотропные $A$-браны. Для этого полезно вспомнить, откуда берется комплекс Флоера, на интуитивном уровне. Рассмотрим пространство гладких путей $P X$ на симплектическом многообразии $X$. Это пространство бесконечномерно, но мы будем обрашаться с ним как с обычным конечномерным гладким многообразием. Из симплектической формы $\omega$ на $X$ мы получаем 1-форму $\alpha$ на $P X$ посредством интегрирования $\omega$ вдоль пути. Точнее говоря, если $\gamma: I \rightarrow X-$ путь на $X$, а $\beta$ - касательный вектор к $P X$ в точке $\gamma$ (т.е. $\beta$-векторное поле, определенное на образе $\gamma$ ), то значение 1 -формы $\alpha$ на векторе $\beta$ определяется как

$$
\int_{I} \omega(\dot{\gamma}(t), \beta(t)) d t
$$

Заметим, что существуют две естественные проекщии из $P X$ на $X$ (начало и конец пути), которые мы обозначим $\pi_{1}$ и $\pi_{2}$. Легко видеть, что $d \alpha=\pi_{2}^{*} \omega-\pi_{1}^{*} \omega$. Таким образом, если мы рассмотрим подмногообразие в $P X$, состояшее из путей, которые начинаются и заканчиваются на каких-нибудь фиксированных лагранжевых подмногообразиях в $X$, то ограничение $\alpha$ на такое подмногообразие будет замкнутым.

Пусть $Y_{1}$ и $Y_{2}$ - два лагранжевых подмногообразия в $X$, и пусть $P X\left(Y_{1}, Y_{2}\right)$ - пространство путей в $X$, начинаюшихся на $Y_{1}$ и кончаюшихся на $Y_{2}$. Тогда оператор $Q=d+2 \pi \alpha$ на пространстве дифференциальных форм на $P X\left(Y_{1}, Y_{2}\right)$ удовлетворяет $Q^{2}=0$, и можно попробовать вычислить его когомологии. (В конечномерном случае 
этот комплекс называется скрученным комплексом де Рама.) Поскольку $P X\left(Y_{1}, Y_{2}\right)$ бесконечномерно, возникают проблемы с точным определением скрученных когомологий де Рама, которые можно преодолевать разными способами. Флоер определил их через теорию Морса. Точнее говоря, если формально применить теорию Морса-Смейла-Новикова-Виттена к вычислению когомологий $Q$, то как раз получится комплекс Флоера.

До сих пор мы игнорировали векторные расслоения $E_{1}$ и $E_{2}$, но этот дефект легко исправить. Рассмотрим векторные расслоения $\pi_{1}^{*} E_{1}^{*}$ и $\pi_{2}^{*} E_{2}$ на $P X\left(Y_{1}, Y_{2}\right)$. У обоих расслоений есть естественные унитарные плоские связности, получаюшиеся как обратньй образ $\nabla_{E_{1}^{*}}$ и $\nabla_{E_{2}}$. Рассмотрим тензорное произведение этих расслоений с плоскими связностями и добавим к связности 1-форму $2 \pi \alpha$. Поскольку $d \alpha=0$, получающаяся связность на $\pi_{1}^{*} E_{1}^{*} \otimes \pi_{2}^{*} E_{2}$ опять плоская, но уже не унитарная. Если мы теперь применим теорию Морса к вьгислению когомологий этого скрученного комплекса де Рама на $P X\left(Y_{1}, Y_{2}\right)$, то получим комплекс Флоера, которьй вычисляет морфизмы между объектами категории Фукая $\left(Y_{1}, E_{1}, \nabla_{E_{1}}\right)$ и $\left(Y_{2}, E_{2}, \nabla_{E_{2}}\right)$.

Вместо пары лагранжевых $A$-бран рассмотрим теперь пару коизотропных $A$-бран. Расслоения $E_{1}$ и $E_{2}$ теперь будут иметь ранг 1. Рассмотрим опять пространство $P X\left(Y_{1}, Y_{2}\right)$, состоящее из путей в $X$, начинающихся на $Y_{1}$ и кончаюшихся на $Y_{2}$. Первая трудность состоит в том, что 1-форма $\alpha$ теперь не замкнута, так что мы не можем использовать ее, чтобы получить скрученный комплекс де Рама. Вторая трудность состоит в том, что связности $\pi_{1}^{*}\left(\nabla_{E_{1}^{*}}\right)$ и $\pi_{2}^{*}\left(\nabla_{E_{2}}\right)$ на расслоениях $\pi_{1}^{*} E_{1}^{*}$ и $\pi_{2}^{*} E_{2}$ тоже не плоские. Как мы сейчас увидим, эти две трудности "компенсируют" друг друга благодаря условиям (б) и (в). Поэтому будем делать то же самое, что и в лагранжевом случае: возьмем тензорное произведение линейных расслоений $\pi_{1}^{*} E_{1}^{*}$ и $\pi_{2}^{*} E_{2}$ и добавим к связности 1 -форму $2 \pi \alpha$. Получившаяся связность не плоская, зато она обладает следуюшим интересньм свойством. Заметим, что поскольку $Y_{1}$ и $Y_{2}-$ многообразия со слоениями, то на $P X\left(Y_{1}, Y_{2}\right)$ тоже имеется естественная структура слоения. Каждьй слой этого слоения состоит из всех путей в $X$, соединяюших фиксированный слой в $Y_{1}$ с фиксированным слоем в $Y_{2}$. Коразмерность этого слоения конечна и равна сумме коразмерностей слоений на $Y_{1}$ и $Y_{2}$. Далее, так как $Y_{1}$ и $Y_{2}$ имеют трансверсальную комплексную структуру, то многообразие $P X\left(Y_{1}, Y_{2}\right)$ со слоением тоже имеет естественную трансверсальную комплексную структуру. Наша связность на расслоении $\pi_{1}^{*} E_{1}^{*} \otimes \pi_{2}^{*} E_{2}$ имеет следующие свойства:

(А) она плоская вдоль слоев слоения;

(Б) в трансверсальных направлениях ее кривизна $\pi_{1}^{*}\left(F_{1}+i \omega\right)-\pi_{2}^{*}\left(F_{2}+i \omega\right)$ имеет тип $(2,0)$.

Отсюда следует, что имеет смысл рассмотреть пучок на $P X\left(Y_{1}, Y_{2}\right)$, состоящий из сечений линейного расслоения $\pi_{1}^{*} E_{1}^{*} \otimes \pi_{2}^{*} E_{2}$, которые локально ковариантно постоянны вдоль слоев слоения и голоморфны в трансверсальных направлениях. Естественно предположить, что когомология этого пучка и дает нам пространство морфизмов между $A$-бранами $\left(Y_{1}, E_{1}, \nabla_{E_{1}}\right)$ и $\left(Y_{2}, E_{2}, \nabla_{E_{2}}\right)$. На формальном уровне это можно показать, рассмотрев квантование топологически скрученной сигма-модели на отрезке с граничньми условиями, соответствуюшими бранам $\left(Y_{1}, E_{1}, \nabla_{E_{1}}\right)$ и $\left(Y_{2}, E_{2}, \nabla_{E_{2}}\right)$. Чтобы получить строгое определение пространства морфизмов, надо найти осмысленную интерпретацию этих формальных конструкций. В случае лагранжевых $A$-бран 
пучок, обсуждавшийся вьше, становится пучком локально ковариантно постоянных сечений плоского расслоения на $P X\left(Y_{1}, Y_{2}\right)$ и его когомологии можно определить через теорию Морса-Смейла-Новикова-Виттена. В общем случае мы не знаем правильной интерпретации.

Трудности, возникающие при попытках обобщить комплекс Флоера на коизотропные $A$-браны, наводят на мысль, что геометрическое описание $A$-бран посредством подмногообразий в $X$ с расслоениями на них в каком-то смысле неправильно. Мы поясним, что мы имеем в виду, посредством следующей аналогии из комплексно-аналитической геометрии. Существует общее понятие голоморфного векторного расслоения на комплексном многообразии, частным случаем которого является понятие голоморфного линейного расслоения. Вместо голоморфного линейного расслоения можно работать с его дивизором, но для расслоений высших рангов такой подход не слишком полезен. Возможно, объекты категории Фукая и коизотропные $A$-браны ранга 1 являются симплектическими аналогами дивизоров, а чтобы получить "правильное" определение $A$-браны, надо найти симплектический аналог понятия голоморфного векторного расслоения (или когерентного пучка). В пользу этой аналогии говорит тот факт, что как описание голоморфного линейного расслоения посредством дивизора, так и описание $A$-браны посредством лагранжева или коизотропного подмногообразия обладают “избыточностью”: линейное расслоение не меняется, если к дивизору добавить дивизор любой мероморфной функции, а $A$-брана не меняется, если подействовать на соответствуюшее подмногообразие потоком любого гамильтонова векторного поля. Мы полагаем, что "правильное" определение категории $A$-бран будет исключительно полезно для понимания зеркальной симметрии, а возможно, и для симплектической геометрии в целом.

\section{СПИСОК ЛИТЕРАТУРЫ}

[1] P.S. Aspinwall, A.E. Lawrence. Derived categories and zero-brane stability // J. High Energy Phys. 2001. № 8, paper 4.

[2] M. F. Atiyah. Topological quantum field theories // Inst. Hautes Études Sci. Publ. Math. 1988. V. 68. P. $175-186$.

[3] А. И. Бондал, М. М. Капранов. Оснащенные триангулированные категории // Матем. сб. 1990. Т. 181. № 5. С. 669-683.

[4] S. Cappell, R. Lee, E. Y. Miller. On the Maslov index // Comm. Pure Appl. Math. 1994. V. 47. № 2. P. 121-186.

[5] D.-E. Diaconescu. Enhanced $D$-brane categories from string field theory // J. High Energy Phys. 2001. №6, paper 16.

[6] R. Dijkgraaf. Intersection theory, integrable hierarchies and topological field theory // New Symmetry Principles in Quantum Field Theory (Cargèse, 1991). New York: Plenum, 1992. P. 95-158. (NATO Adv. Sci. Inst. Ser. B Phys. V. 295.)

[7] M.R. Douglas. $D$-branes, categories and $N=1$ supersymmetry // J. Math. Phys. 2001. V. 42. № 7. P. 2818-2843.

[8] K. Fukaya. Morse Homotopy, $A_{\infty}$-category and Floer Homologies // Preprint № 020-94. Berkley: MSRI, 1994.

[9] K. Fukaya, Y-G. Oh, H. Ohta, K. Ono. Lagrangian intersection Floer theory-anomaly and obstruction // Preprint; http://www.kusm.kyoto-u.ac.jp/ fukaya/fukaya.html.

[10] K. Gawȩdzki. Lectures On conformal field theory // Quantum Fields and Strings: A Course for Mathematicians. V. 1, 2 (Princeton, NJ, 1996/1997). Providence, RI: Amer. Math. Soc., 1999. P. 727-805. 
[11] И. М. Гельфанд, И.Я. Дорфман. Скобка Схоутена и гамильтоновы операторы // Функц. анализ и его прил. 1980. Т. 14. № 3. С. 71-74.

[12] С. И. Гельфанд, Ю.И. Манин. Методы гомологической алгебры. Т. 1. Введение в теорию когомологий и производные категории. М.: Наука, 1988.

[13] V. Golyshev, V. Lunts, D. Orlov. Mirror symmetry for abelian varieties // J. Algebraic Geom. 2001. V. 10. № 3. P. 433-496.

[14] B. R. Greene, M. R. Plesser. Duality in Calabi-Yau moduli space // Nuclear Phys. B. 1990. V. 338. № 1. P. 15-37.

[15] R. Hartshorne. Residues and Duality. Berlin: Springer-Verlag, 1966. (Lecture Notes in Math. V. 20.)

[16] R. T. Hoobler. Brauer groups of abelian schemes // Ann. Sci. École Norm. Sup. (4). 1972. V. 5. P. $45-70$.

[17] K. Hori, S. Katz, A. Klemm, R. Pandharipande, R. Thomas, C. Vafa, R. Vakil, E. Zaslow. Mirror Symmetry. Providence, RI / Cambridge, MA: Amer. Math. Soc. / Clay Mathematics Inst., 2003. (Clay Math. Monogr. V. 1.)

[18] A. Kapustin. Topological strings on noncommutative manifolds // Int. J. Geom. Methods Mod. Phys. 2004. V. 1. №1-2. P. 49-81.

[19] A. Kapustin, D. Orlov. Vertex algebras, mirror symmetry, and D-branes: The case of complex tori // Comm. Math. Phys. 2003. V. 233. № 1. P. 79-136.

[20] A. Kapustin, D. Orlov. Remarks on A-branes, mirror symmetry, and the Fukaya category // J. Geom. Phys. 2003. V. 48. №1. P. 84-99.

[21] М. Касивара, П. Шапира. Пучки на многообразиях. М.: Мир, 1997.

[22] S. Katz, E. Sharpe. D-branes, open string vertex operators, and Ext groups // Adv. Theor. Math. Phys. 2002. V. 6. №6. P. 979-1030.

[23] B. Keller. Derived categories and their uses // Handbook of Algebra / ed. M. Hazewinkel. V. 1. Amsterdam: North-Holland, 1996. P. 671-701.

[24] B. Keller. Introduction to $A_{\infty}$-infinity algebras and modules // Homology Homotopy Appl. 2001. V. 3. № 1. P. 1-35 (electronic).

[25] M. Kontsevich. Homological algebra of mirror symmetry // Proceedings of International Congress of Mathematicians (Zurich, 1994). Basel: Birkhäuser, 1995. P. 120-139.

[26] M. Kontsevich, Y. Soibelman. Homological mirror symmetry and torus fibrations // Symplectic Geometry and Mirror Symmetry (Seoul, 2000). River Edge, NJ: World Scientific, 2001. P. 203-263.

[27] C. I. Lazaroiu. On the structure of open-closed topological field theory in two dimensions // Nuclear Phys. B. 2001. V. 603. № 3. P. 497-530.

[28] C. I. Lazaroiu. Unitarity, D-brane dynamics and D-brane categories // J. High Energy Phys. 2001. № 12 , paper 31.

[29] W. Lerche, C. Vafa, N.P. Warner. Chiral rings in $N=2$ superconformal theories // Nuclear Phys. B. 1989. V. 324. № 2. P. 427-474.

[30] Y. Li. Anomalies and graded coisotropic branes // arXiv:hep-th/0405280.

[31] G. Moore. D-branes, RR-fields and K-theory // The Duality Workshop (2001); http: //online.itp.ucsb.edu/online/mp01.

[32] S. Mukai. Duality between $D(X)$ and $D(\widehat{X})$ with its application to Picard sheaves // Nagoya Math. J. 1981. V. 81. P. 153-175.

[33] D.O. Orlov. Equivalences of derived categories and $K 3$ surfaces // J. Math. Sci. (New York). 1997. V. 84. № 5. P. 1361-1381.

[34] Д. О. Орлов. Производные категории когерентных пучков на абелевых многообразиях и эквивалентности между ними // Изв. РАН. Сер. матем. 2002. Т. 66. № 3. С. 131-158.

[35] Д. О. Орлов. Производные категории когерентных пучков и эквивалентности между ними // УМH. 2003. T. 58. № 3. C. 89-172.

[36] J. Polchinski. String Theory. V. 2: Superstring Theory and Beyond. Cambridge: Cambridge Univ. Press, 1998.

[37] J. Polchinski. Dirichlet-branes and Ramond-Ramond charges // Phys. Rev. Lett. 1995. V. 75. № 26. P. 4724-4727. 
[38] A. Polishchuk. Symplectic biextensions and generalization of the Fourier-Mukai transforms // Math. Res. Lett. 1996. V. 3. №6. P. 813-828.

[39] A. Polishchuk, E. Zaslow. Categorical mirror symmetry: The elliptic curve // Adv. Theor. Math. Phys. 1998. V. 2. № 2. P. 443-470.

[40] G. B. Segal. The definition of conformal field theory // Differential Geometrical Methods in Theoretical Physics (Como, 1987). Dordrecht: Kluwer, 1988. P. 165-171. (NATO Adv. Sci. Inst. Ser. C Math. Phys. Sci. V. 250.)

[41] A. Strominger, S. T. Yau, E. Zaslow. Mirror symmetry is T-duality // Nuclear Phys. B. 1996. V. 479. № 1-2. P. 243-259.

[42] R. G. Swan. Hochschild cohomology of quasiprojective schemes // J. Pure Appl. Algebra. 1996. V. 110. № 1. P. 57-80.

[43] J.L. Verdier. Catégories dérivées // Cohomologie Étale. Séminaire de Géométrie Algébrique du Bois-Marie SGA $4 \frac{1}{2}$. Berlin: Springer-Verlag, 1977. P. 262-311. (Lecture Notes in Math. V. 569.)

[44] E. Witten. Phases of $N=2$ theories in two dimensions // Nucl. Phys. B. 1993. V. 403. № 1-2. P. 159-222.

[45] E. Witten. Topological sigma models // Comm. Math. Phys. 1988. V. 118. № 3. P. 411-449.

[46] E. Witten. Mirror manifolds and topological field theory // Essays on Mirror Manifolds. Hong Kong: Internat. Press, 1992. P. 120-158.

[47] E. Witten. Chern-Simons gauge theory as a string theory // The Floer Memorial Volume. Basel: Birkhaüser, 1995. P. 637-678. (Progr. Math. V. 133.)

Калифорнийский технологический институт,

Поступила в редакцию

Пасадена, Калифорния, США;

05.08 .2004

Математический институт им. В. А. Стеклова РАН, Россия

E-mail: kapustin@theory.caltech.edu, orlov@mi.ras.ru 